\title{
EVOLUCIÓN Y RELACIONES FILOGENÉTICAS ENTRE LOS GRUPOS DE MAYOR RANGO TAXONÓMICO DE ATÍRIDOS Y OTROS "BRAQUIÓPODOS ARTICULADOS"
}

\author{
Fernando ÁLVAREZ' y Sandra CARLSON \\ ' Departamento de Geología. Universidad de Oviedo, c/ Jesús Arias de \\ Velasco, s/n, 33005 Oviedo, España. Correo electrónico: \\ fernando@asturias.geol.uniovi.es \\ ? Department of Geology. University of California, Davis, California 95616. \\ USA. Correo electrónico: sjcarlson@ucdavis. edu
}

\begin{abstract}
Álvarez, F. y Carlson, S.J. 1998. Evolución y relaciones filogenéticas entre los grupos de mayor rango taxonómico de atíridos y otros "braquiópodos articulados". [Evolution and phylogenetic relationships among athyridide higher taxa and other "articulate brachiopods"]. Revista Española de Paleontología, 13 (2), $209-234$. ISSN 0213-6937.
\end{abstract}

\begin{abstract}
Phylogenetic relationships among higher athyridide taxa and of spire-bearing brachiopods in general, are not universally agreed because of the complex pattern of character evolution within the group. Our results, using phylogenetic methodology, in conjunction with traditional methods, showed a more complete and more easily testable picture of athyridide evolution than either method used alone. The results of eight experimental phylogenetic analyses using outgroup criteria for polarity determination are compared and contrasted, morphological character evolution within and between groups is discussed and comparisons of stratigraphical and outgroup methods of polarity determination are presented. Cladistic analysis suggests that "spiriferides" are a group separate from atrypides and athyridides; koninckinidines, meristelloids, athyridoids, retziidines, retzielloids and atrypides appear as well defined, consistent clades; the Dayiinae consistently clusters with the lissatrypidines and the athyrisinins with the athyridides; the retziidines appear as the most derived group. Agreement between the stratigraphical first appearances of the main athyridide groups and their cladistic rank indicates a similar direction of character polarity in the evolution of the athyridides. According to the results of the analyses, several shell structures evolved independently in different groups of "articulate brachiopods".
\end{abstract}

Keywords: "Articulate brachiopods", "spirebearers", athyridides, phylogenetic analyses, evolution.

\section{RESUMEN}

Es difícil lograr un acuerdo sobre un modelo de relaciones filogenéticas entre los grupos de mayor rango taxonómico de atíridos y de los braquiópodos con braquidio espiralado, en general, debido a la complejidad de la evolución de los caracteres dentro del grupo. Los resultados obtenidos en este estudio, utilizando una metodología filogenética junto con los métodos tradicionales, mostraron una imagen más completa y fácilmente comprobable, de la evolución de los atíridos, que cualquiera de ellos utilizado independientemente. Los resultados de ocho análisis filogenéticos utilizando grupos externos para la determinación de las polaridades son comparados y contrastados, se discute la evolución de los caracteres morfológicos dentro y entre los grupos, y se realiza una comparación entre los métodos estratigráficos y de grupo externo para la determinación de las polaridades. El análisis cladístico sugiere que los "espiriféridos" forman un grupo separado de los atrípidos y los atíridos; koninckinidinos, meristelloideos, atiridoideos, retziidinos, retzielloideos y atrípidos aparecen como clados consistentes, bien definidos; los Dayiinae se agrupan reiteradamente con los lissatripidinos, y los atirisininos, con los atirididos; los retziidinos aparecen como el grupo más derivado. La concordancia entre los datos de primera aparición de los grupos principales de atíridos y su rango cladístico sugiere que ambos tipos de análisis indican una dirección similar en la evolución de los caracteres en los atíridos. De acuerdo con los resultados de los análisis, diversas estructuras de la concha surgieron independientemente en diferentes grupos de "braquiópodos articulados".

Palabras clave: "Braquiópodos articulados", espiralio, atíridos, filogenia, evolución. 


\section{INTRODUCCIÓN}

Desde 1883, en que Waagen erigió el suborden Helicopegmata o Spiriferacea para incluir en él aquellos braquiópodos que desarrollaron braquidio espiralado (los Spiriferidae, Athyridae, Nucleospiridae y Atrypidae de Davidson, 1881), su origen, posición sistemática y relaciones filogenéticas fueron, y aún siguen siendo, objeto de discusión. La mayoría de autores mantuvieron la clasificación de Waagen, si bien, cambiando el rango o la relación entre los grupos por él propuestos (e.g. Hall y Clarke, 1894; Schuchert, 1896, 1929; Zittel, 1903; MuirWood, 1955; Ivanova, 1960; Boucot et al., 1965; Grunt, 1986, 1989; Harper et al., 1993; Carter et al., 1994; Copper y Gourvennec, 1996; Dagis, 1996a, b; Williams et al., 1996). Frecuentemente, la clasificación de los "braquiópodos articulados" se basó en uno, o unos pocos, caracteres morfológicos aislados (e.g. condición estrófica o astrófica de la concha, presencia o ausencia de puntos o pseudopuntos, tipo de braquidio, etc.). Construir un sistema filogenético basándose sólo en un carácter es peligroso, por importante o útil que éste sea o parezca ser, ya que muchas semejanzas morfológicas son fruto de evoluciones paralelas o convergentes no representando, por tanto, una verdadera relación filogenética. La unión de todos los grupos de braquiópodos con espiralio en el orden Spiriferida (sensu Boucot et al., 1965), se basó principalmente, si no de modo exclusivo, en la presencia de este carácter. Sin embargo, aunque este tipo de braquidio es característico de estos grupos, la calcificación de un espiralio no indica necesariamente una relación directa entre ellos, ya que este fenómeno bien pudo haberse producido en diferente líneas filéticas (hecho ya sugerido por Thomson, 1925; Ivanova, 1959; Rudwick, 1970; Wright, 1979a, b; Carter et al., 1994, etc.). Parece razonable admitir un posible origen múltiple para muchas de las características morfológicas y funcionales más importantes observadas en los braquiópodos (ver también Rudwick, 1970). Se es consciente también de que algunas categorías sistemáticas pueden abarcar diferentes líneas filogenéticas, ya que muchas características morfológicas (e.g. presencia de ornamentación radial, desarrollo de interárea o palintropo, lamelas dentales, soportes para el pedúnculo, tipo de cardinalio, espiralio doble ... ) pueden aparecer, independientemente, en líneas filéticas diferentes, provocando numerosos casos de homeomorfía. Por lo tanto, al separar categorías sistemáticas, debe considerarse importante no sólo la existencia de espiralio (o por ejemplo, la presencia de puntos o pseudopuntos), sino también otras peculiaridades de la concha, tales como su forma y tamaño, presencia y tipo de ornamentación radial, tipo de plegamiento, área cardinal (palintropo), deltirio, nototirio, plataformas internas, proceso cardinal, etc. De una semejanza estructural global, puede deducirse convergencia o paralelismo así como un linaje común; estos dos tipos de semejanza deben diferenciarse, lo que no resulta siempre fácil.

En el presente trabajo se intenta una aproximación filogenética (cladística) (ver Wiley, 1981; Forey, 1987; Fortey y Chatterton, 1988; Carlson, 1991, 1993, 1995;
Holmer et al., 1995; Williams et al., 1996) a los braquiópodos con braquidio espiralado, considerando el mayor número posible de caracteres externos e internos, teniendo en cuenta su desarrollo tanto ontogenético como filogenético, y tratando de evitar la tendencia a dar indiscriminadamente peso taxonómico a ciertos caracteres que pudiesen ser considerados como la panacea para la clasificación sistemática. Se espera que la hipótesis de trabajo que aquí se presenta, refleje la filogenia de los braquiópodos con braquidio espiralado en general y la de los atíridos, en mayor detalle. En el intento se encontraron algunas limitaciones, como son la escasez de datos referentes al desarrollo ontogenético de los taxones estudiados (algunos basados sólo en unos pocos ejemplares o incluso sólo en el tipo) y de la microstructura y crecimiento de las estructuras esqueléticas, un conocimiento parcial (a veces equívoco) de su distribución estratigráfica y geográfica, y lo que es muy importante en los grupos aquí considerados, la ausencia de descripción de las características de la microornamentación externa y de la morfología interna (braquidio, espiralio y aparato yugal) presente en muchos géneros. Tras el estudio detallado de la estructura de la concha y, en algunos casos, del afortunado descubrimiento de espiralio, algunas especies consideradas tradicionalmente como órtidos, estrofoménidos o rinconélidos son ahora consideradas espiriféridos sensu lato (e.g. Williams y Hurst, 1977; Williams y Brunton, 1993; Copper, 1996). Por contra, grupos considerados espiriféridos s.l., como por ejemplo los leptocoélidos, son ahora considerados rinconélidos debido a su morfología general y a la ausencia de un espiralio calcificado (ver Cocks, 1978).

En el presente trabajo se es consciente también de que el modelo de distribución de los caracteres tenidos por primitivos o por derivados es muy complejo, tanto temporal como taxonómicamente. Grupos de "braquiópodos articulados" que pudiesen ser considerados primitivos, debido a su temprana aparición estratigráfica (e.g. los pentaméridos), se encuentran, sin embargo, entre los más derivados respecto al resto de grupos de "articulados" y a los "inarticulados" utilizados como grupo externo en los análisis filogenéticos de Carlson (1991). Por el contrario, espiriféridos estróficos, relativamente recientes en su aparición en el registro estratigráfico, forman un grupo parafilético, pobremente definido, en la base de los dos clados mayores de "articulados" (Carlson, 1991: p. 8, Fig. 4).

En la redacción de este trabajo se ha seguido, en lo posible, el glosario español de términos utilizados en sistemática cladística elaborado por Sanchiz y Valdecasas (1980).

Figura 1. Cladograma número uno (de los 186 igualmente más parsimoniosos resultantes en el primer análisis). Se representa la distribución estratigráfica conocida para cada taxón mediante barras. 




\section{MÉTODOS}

Las relaciones filogenéticas entre los atíridos s.l. y otros "braquiópodos articulados" fueron estudiadas utilizando métodos en los que la determinación de la polaridad de las transformaciones viene dada por las características de un grupo externo tenido por ancestral al grupo bajo estudio. Los análisis fueron realizados utilizando el programa PAUP 3.0q para Apple Macintosh (Swofford, 1990), complementado con el programa MacClade 3.0 (Maddison y Maddison, 1992). Detalles sobre la metodología filogenética han sido explicados por Carlson (1991, 1993).

\section{TAXONES}

Para que el número de taxones fuese razonablemente manejable, fue necesario seleccionar sólo uno o dos géneros en representación de cada grupo principal escogido por Carlson (1991) para su análisis filogenético de las superfamilias de "braquiópodos articulados". Así, en un primer análisis fueron incluidos en la matriz 55 géneros (grupo interno): cuatro rinconélidos primitivos (Ancistrorhyncha, Dorytreta, Rostricellula, Trigonirhynchia); dos pentaméridos (Stenocamara, Xenelasma); cinco "espiriféridos" (Cyrtia, Eospirifer, Kozlowskiellina, Martinia y Syringothyris); cuatro braquiópodos astróficos, con la concha semejante a la de los rinconélidos (de los cuales probablemente hayan derivado paedomórficamente) y que aunque primitivos, desarrollaron un braquidio espiralado más o menos complejo (tres anazigidinos: Idiospira, Manespira y Protozyga y un zigospírido: Zygospira); Lissatrypa junto con otros 5 atrípidos costados (Atrypa, Eifelatrypa, Independatrypa, Pseudoatrypa y Zygospiraella), dos koninckinoideos (Amphiclina y Koninckina), tres retziéllidos (Metathyrisina, Molongia y Retziella), siete meristelloideos (Cryptothyrella, Dicamaropsis, Hindella, Hyattidina, Merista, Rowleyella y Whitfieldella), cuatro retziidinos (Cassianospira, Eumetria, Hustedia y Neoretzia), doce atiridoideos (Athyris, Athyrisina, Didymothyris, Diplospirella, Glassina, Helenathyris, Hexarhytis, Ochotathyris, Pachyplax, Pexidella, Plicathyris y Spirigerella) más Nucleospira y tres géneros de controvertida situación filogenética (Bifida, Dayia y Kayseria). Dos terebratúlidos, el terebratellidino Cryptonella y el terebratulidino Dielasma, fueron también incluidos en la matriz (grupo interno).

Para investigar en mayor detalle las relaciones filogenéticas entre aquellos grupos considerados tradicionalmente como atíridos, el número de géneros pertenecientes a cada uno de esos grupos (retziidinos, meristelloideos y atiridoideos) fue sucesivamente incrementado en el grupo interno. Desde la publicación de los dos volúmenes del Treatise on Invertebrate Paleontology (Williams et al., 1965) el número de géneros de atíridos ha duplicado con creces su número (comparar e.g. Boucot et al., 1965 con Álvarez et al., 1994). Desafortunadamente, el interior de un considerable número de géneros (y en ocasiones incluso superfamilias) se conoce muy poco, o se desconoce por completo. La inclusión (a veces forzosa) de taxones con una cantidad significativa de caracteres morfológicos desconocidos, frecuentemente conduce a un gran incremento en el número de soluciones igualmente "parsimoniosas". Para que el número de taxones siguiese siendo razonablemente manejable a la vez que se intentaba evitar el colapso de los árboles en politomías (e.g. Smith, 1994), en los sucesivos análisis fueron introducidos aquellos géneros mejor conocidos (externa e internamente), representativos de las familias y subfamilias actualmente en uso (ver Álvarez et al., en prensa).

Al seleccionar los taxones para ser incluidos en la matriz, ha de tenerse en cuenta también que la inclusión o la eliminación de uno solo puede tener una gran influencia en el análisis y en la topología o morfología del árbol resultante. Este efecto puede verse magnificado en el caso de que el taxón incluido o eliminado sea considerado normalmente ya como "problemático". En este caso, las afinidades filogenéticas varían significativamente.

Para designar los diferentes grupos de braquiópodos actualmente clasificados en distintos taxones de rango alto, se utilizaron, a veces, nombres informales (e.g. atíridos por Athyridida, porambonítidos por Porambonitidae, etc.). Las relaciones filogenéticas, rango taxonómico relativo y clasificación a un nivel menor de estos taxones están siendo evaluados en la actualidad. Estos nombres informales permiten establecer relaciones entre los braquiópodos sin hacer demasiado énfasis en el rango o definición actual del nombre en cuestión. Siguiendo a Gauthier (1986), taxones dudosos o identificados como parafiléticos se mantienen siempre entre comillas.

\section{CARACTERES}

Se recopiló información sobre 83 caracteres morfológicos relacionados con la concha [incluyendo la forma y ornamentación de las valvas, interiores dorsal y ventral (especialmente cardinalio y espiralio) y su estructura] (Tablas 1 y 2 ). Se reconocieron estados binarios o múltiples en los caracteres. No se asignó un orden a la aparición de los distintos caracteres (o de los distintos estados de los mismos), de forma que la dirección en que se produjesen las transformaciones de los mismos viniese polarizada por las características morfológicas de los grupos tenidos por primitivos o ancestrales (grupo externo). Ninguna transformación fue considerada como "irreversible". En 6 de los 8 análisis realizados (todos ellos descritos en el apartado de resultados) se dio el mismo peso a todos los caracteres. Dos caracteres (los números 55 y 56 ) fueron pesados 10 veces más que los otros en el análisis 6 , y los números 77 y 80 en el análisis 8.

\section{PROCEDIMIENTO}

Se realizó una amplia experimentación con diferentes grupos externos e internos y las distintas rutinas que permite el programa, para así evitar la aceptación acrítica del resultado primero o de aquel que pareciese más 
Carácter

1. Tamaño

2. Forma

3. Contorno (vista dorsal)

4. Margen anterior

5. Contorno (vista lateral)

6. Perfil lateral

7. Grado de convexidad (concha adulta)

8. Convexidad (concha adulta)

9. Prominencia gancho ventral

10. Curvatura umbo valva ventral

11. Ornamentación radial

12. Si costillas

13. Si otros

14. Espinas

15. Distribución de la ornamentación. radial

16. Líneas de crecimiento

17. Líneas de crecimiento (espaciado )

18. Lamelas

19. Pliegue medio y seno

20. Pliegue medio y seno (ornamentación)

21. Si costados

22. Anchura de pliegue y seno

23. Longitud de pliegue y seno

24. Plegamiento

25. Si mixto

26. Area cardinal ventral (palintropo)

27. Orientación del área

28. Area cardinal dorsal

29. Orientación del área

30. Línea de charnela

31. Si astrófica

32. Anchura de la línea de charnela

33. Apertura peduncular

34. Si deltirio

35. Si foramen

36. Si foramen + deltirio, deltirio

37. Si foramen + deltirio, foramen

38. Soporte peduncular

39. Si soporte peduncular

40. Tamaño de los dientes

41. Lamelas dentales

42. Espesor lamelas dentales

43. Longitud lamelas dentales

44. Si largas

45. Orientación de las lamelas dentales

46. Placas mistroquiales

47. Shoe-lifter ventral

48. Posición del Shoe-lifter ventral

49. Espondilio

50. Espondilio soportado por

51. Septo medio ventral

52. Si largo y relacionado con espondilio

53. Huellas musculares ventrales

54. Plataforma cardinal

55. Si presente

56. Proceso cardinal

57. Miofragma

58. Septo medio dorsal

59. Longitud del septo medio dorsal

60. Altura del septo medio dorsal

61. Septalio

62. Si presente

63. Longitud del septalio

64. Tipo se septalio

65. Placas cubriendo el septalio

66. Cruralio

67. Shoe-lifter dorsal

68. Braquidio

69. Si espiralio, ápices dirigidos

70. Lamela primaria

71. Yugo

72. Si presente o incompleto

73. Ramas laterales del yugo comenzando

74. Ramas laterales, orientación

75. Arco yugal

76. Visera yugal

77. Tallo yugal

78. Tallo yugal, orientación

79. Bifurcaciones yugales (arms)

80. Lamelas yugales accesorias

81. Lamelas yugales accesorias libres

82. Estructura de la concha

83. Capa prismática

\section{Estado}

Pequeño (0); moderado (1); grande (2); muy grande (3)

$\mathrm{L}=\mathrm{A}(0) ; \mathrm{L}>\mathrm{A}(1) ; \mathrm{L}<\mathrm{A}(2) ; \mathrm{L}=\mathrm{A} \circ \mathrm{L}>\mathrm{A}(3) ; \mathrm{L}=\mathrm{A} \circ \mathrm{L}<\mathrm{A}(4) ; \mathrm{L}=\mathrm{A}, \mathrm{L}>\mathrm{A} \circ \mathrm{L}<\mathrm{A}(5)$

Subtriangular (0); subpentagonal (1); subcircular (2); subelíptico (3); romboidal (4)

No lobado (0); lobado (1); denticulado (2)

Comprimido posteriormente (0); elíptico (1); comprimido anteriormente (2)

Biconvexo (0); plano o concavoconvexo (1)

Débil (0); moderada (1); fuerte (2); muy fuerte (3)

VD $>V V(0)$; VD<VV (1); VD=VV (2)

VV no rostrada (0); moderadamente (1); fuertemente (2); muy fuertemente rostrada (3)

Recto (0); moderada (1); fuerte (2); muy fuerte (3)

Ausente (0); pliegues (1); costillas (2); líneas finas (3); otras (4)

15 ó $<15(0) ;>15(1)$

Líneas bifurcantes (0); en "espina de pescado" (1); en "zigzag" (2)

Ausentes (0); sólidas redondeadas (1); sólidas tabulares (2); huecas (3); muy finas (4)

Toda la concha ( 0$) ; 2 / 3$ anteriores (1); $1 / 3$ anterior $(2) ; 2 / 3$ posteriores (3)

Ausentes (0); débiles (1); fuertes (2)

Próximas (0); distantes (1)

Ausentes (0); cortas (1); largas (2); muy largas (3); muy gruesas (4)

Ausente (0); débil (1); fuerte (2); pliegue en ambas valvas (3); sulco en ambas valvas (4)

Lisos (0); costados (1); radial fina (2); líneas finas, bifurcantes (3)

Similar a la ornamentación en los flancos (0); diferente (1)

Ancho (0); estrecho (1)

Toda la concha (0); $2 / 3$ anteriores (1); $1 / 3$ anterior (2)

Alterno (0); opuesto (1); mixto (2)

Bien desarrollado (0); moderado (1)

Rudimentaria(0): reducida (1); moderada (2); extensa (3)

Cata-apsaclina (0); apsa.(1); apsa-orto.(2); ortoclina(3); orto-anaclina (4)

Rudimentaria( 0$)$ : reducida (1); extensa (2)

Apsaclina (0); anaclina(1); ortoclina (2)

Estrófica (0); astrófica(1)

Casi estrófica (0); claramente astrófica (1)

Corta (0); media (<máx. anchura concha) (1); larga (= máx. anch.) (2)

Ausente (0); deltirio (1); foramen (2); foramen + deltirio (3)

Abierto (0); parcialmente cubierto (1); cubierto totalmente (2)

En posición submesotírida (0); meso-permeso. (1); epitírida (2)

Abierto (0); parcialmente cubierto (1); cubierto totalmente (2)

Posición submesotírida(0); meso-permeso.(1); epi.(2); permeso-epi.(3)

Ausente (0); presente (1)

Collar peduncular simple (0); complejo (1); fulcrum (2); placa deltirial (3); otros (4)

Pequeño (0); medio (1); grande (2)

Indistintas (0); distintivas (1); ausentes (2)

Delgadas (0); moderadas (1); gruesas (2)

Cortas (0); medias (1); largas (2)

Extendiéndose a lo largo de las huellas musculares (0); no extendiéndose (1)

Subparalelas (0); convergen dorsalmente (1); divergen dorsalmente (2); cóncavas (3)

Ausentes (0); presentes (1).

Ausente (0); presente (1).

Entre las lamelas dentales ( 0$)$; soportando las lamelas dentales (1).

Ausente (0); presente (1).

No septo (sésil) (0); septo bajo y ancho (1); septo bajo y delgado (2); septo alto (3)

Ausente (0); corto (1); largo, soportando al espondilio en toda su longitud (2); largo, pero no relacionado con un espondilio (3).

Se extiende anteriormente más que el espondilio (0); no (1)

Muy impresas (0); moderadas (1); débiles (2)

Ausente (0); presente (1)

Disjunta (0); perforada apicalmente (1); no perforada (2)

Ausente (0); rudimentario (1); moderado (2); muy desarrollado (3); otros (4)

Ausente (0); presente (1)

Ausente (0); presente (1)

Corto (0); largo (1)

Moderada (0); muy alto (1)

Ausente (0); presente (1)

Poco profundo (0); profundo y estrecho (1); profundo y ancho (2)

Corto (0); largo (1)

Sin soporte (0); soportado por un septo medio (1); sésil (2)

Ausentes (0); parcialmente cubierto (1); totalmente cubierto (2)

Ausente (0); presente (1)

Ausente (0); presente (1)

Ausente (0); presente (espiralio) (1); en forma de rizo (2).

Lateralmente (0); medio (1); dorsal (2); ventral (3); dorsomedianamente (4); ventrolateralmente (5)

Prolongación directa de los crura (0); curvando lateralmente de los crura (1); curvando posterodorsalmente desde los crura (2).

Ausente (0); presente (1); incompleto (2).

Situado dorsalmente $(0)$; situado ventralmente (1)

Mitad longitud VD (0); posterior a ella (1); anterior (2)

Verticales (0); inclinadas anteriormente (2); posteriormente (3); casi paralelas al plano comisural (4)

Agudo (0); redondeado (1); otro (2)

Ausente (0); presente y anterior (1); posterior(2).

Ausente (0); presente y corto (1); largo (2).

Vertical (0); inclinado moderadamente en dirección posterior (1); fuertemente inclinado post. (2); horizontal (3); anteriormente inclinado (4)

Ausentes (0); presentes (1)

Ausentes (0); libres (1); vueltas a unir al tallo (2); unidas a las ramas laterales del yugo (3); unidas secundariamente al espiralio (4)

Cortas $(0)$; terminando en las proximidades del inicio de las ramas laterales (1); intercalando con los conos principales hasta el ápice (2)

Sin puntos (0); con puntos (1)

Siempre presente (0); a veces (1); nunca presente (2)

Tabla 1. Lista de caracteres utilizados para construir la matriz (ver Tabla 2). Los taxones utilizados en cada análisis, así como el tipo (ordenado o no) y el peso de los caracteres, se indica en el texto, en el apartado <Métodos $>$. 


Acambona
Amphiclina
Anathyris
Ancistrorhyncha
Athyris
Athyrisina
Atrypa
Bifida
Billingsella
Buchanathyris
Camarium
Camarophorella
Camarospira
Cassianospira
Charionella
Charionoides
Clavigera
Cleiothyridina
Collarothyris
Composita
Cryptonella
Cryptospirifer
Cryptothyrella
Cyrtia
Dalmanella
Dayia
Dicamara
Dicamaropsis
Didymothy

213010122221 NO0100110020N220N1 103NN2110? ONNNNNONONON2?????????????? 10???????????? 12

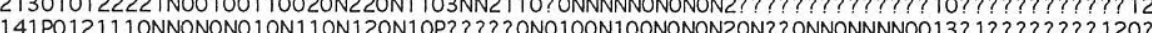
32311012111 NNOO20220NOO2033101023 NNO10N1121000ONONON211210NNONNNNO0 1021111112011102 123010121121 NOOONO110020N120N11010NNNONO101 NOOONONON2100OONNONNNNOOONNNNNNNNNNNNNO2 24301010110 NNON20110N11 ON1 1 ON1 103NNO1ON1 101N3OONONON211210NNONNNNO0 1021100112111102 253010221221 NOO202210100N12101113NNO10N1 101 NOOONONON211?? ONNONNNNO01021111112111002 253010221221 NOO2O2210100N121011 13NNO10N1 101 NOOONONON2 11 ?? ONNONNNNO01021111112111002 033012111120 NOO201111100N120N1103NNO? ON12NNNNOONON3N1 ON401110NNNNO01521110201110NO2 131010010021 NOO1 POONNNN1 N3221 ON21 2NNNON11 PPN10ONONON1ON4? ONNONNNNOOONNNNNNNNNNNNNO2 11301011220 NNON1?21 ONP2ON12ON1 103NNO1 ONO 101 N10ONONON21110ONNONNNNO01021100101300NO2 11301012220 NNON10010N120N120N1 103NNO10N0111020110NONNONO01111011000????????????0? 15301022220 NNON100ONNNN1 N130N1 103NNO10N01121210N1120NONO0111121100110211210P2113NO2

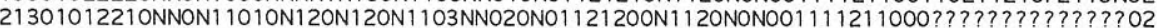
01101011301 NNOO??0411101N330N1023NN20ONO2NNNNNONONON212301110NNNNO01021111102414N12 11301022220 NNON10010N120N120N1103NNO10NOONONOOONONONOONOOONN12120001021102002112NO2 11301022220 NNON10010N120N120N1 103NNO2ONO110NOOONONON10N001101211000??????????????02 $323 P 1012111$ NNOO1? 140NOO1N3310ON23NNO10N1 111 N2OONON1 NO 12310NNONNNNOO 102111 $11 ? 211100 ?$ 2530101211 ONN2N2O210NO1ON120N1 103NNO1ON110ONPOONONON11121ONNONNNNO01021110112111102 153? 2021210NNON1 1010N1 10N220N1 101 1NNN130110N10ONONON2111PONNONNNNO01021111112111102 P3301022220NNON10110NO10N120N1103NN010N1101N10ONONON21230ONNONNNN001021110112011102

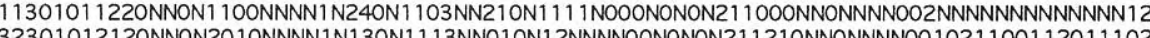
21301022230 NNON1 1010NO2ON120N1 103NNO2ONO112000ONONONOONO?? 1111111001021112002100 NO0 12302231000 NNON1?010NOOON30120N212NNNONO 111 NOOONONON2ON4? ONNONNNNOO100ONNNNNNNNNNO2 112010110021 NOO 11011 OP2ON22210N210NNNON1?????OONONONOON4? ONNONNNNOOONNNNNNNNNNNNN12 01102231130 NNONONO 1 ONO1ON14ON1 10? ONNNONO2NNNNOONONON1 $10 ? 011$ 10ONNNNO01511024001200NO2

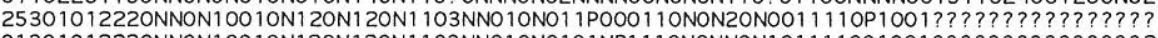

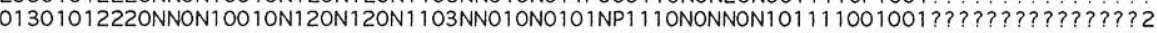
13312021210 NNON1 1010N1 10N220N1 1011 NNN120110N10ONONON2110PONNONNNNO01021111112111102 21301011230 NNONONO1ONP1 ON240N1 103NN22101111 NOOONONON2ON4OONN12120002NNNNNNNNNNNNN12 01301012110 NNON1 10ONNNN1N130N1 103NNO1ONO100N2OONONON212210NNONNNN001021111102111202 010010021120 NOOONO? 1010 ? N130N1101? NNNONO 101 NOOONONON21000ONNONNNNOOONNNNNNNNNNNNNO2 222023001021 NOO??? ONNNN1 N210N? 013NN200N12NNNNOONONON? 101 ? ONNONNNNO012121 NNNNNNNNNO2 223010221121 NOO 1 ? 0110000 N21 120N21 1 NNNON? 111 NOOONONON2ON4? ONNONNNNOO 1000 NNNNNNNNNNO2 213010122221 NOO 100110020 N22ON1103NN21POO2NNNNNONONON2123OONNONNNNO01021111102110N12 11111011120 NNON10010N120N120N1103NNO 10NO10ONOOONONON211? OONNONNNNO010211 10 ? 00NO 1102 00201002010 NN4ON? 1ONNNN1N130N1 103NN? ? ONO10ONOOONONON211000NNONNNNO0 10211111?2111202 $2331102 P 221$ NNOO20220NOO2022101003NNO10N1 100N100NONON211210NNONNNNO01021111112011102 24301011220 NNON210ONNNN1 N12ON1103NNO10NO1120000NONON2ONO1ONN110PO001021123101200N?2 211010121221 NO0110111010N1 10N1103NNP10N12NNNNNONONON212101010NNNNO01021100120NNNN12 111010121221 NO0210111010N1 10N1 113NN210N12NNNNNONONON21000ONOONNNNOO?????????????12 01301011301 NNOO??0411101N33ON1023NN210NO2NNNNNONONON212301110NNNNO01021110102111212 113010222120 NO01? 0110021 N23ON1013NN21 1002NNNNNONONON212201000NNNNO0102111110210NN12 013010122120 NOO? ? OONNNN1N230N1003NN211002NNNNNONONON212201000NNNNO0102110010110NN12 13101022220 NNON10020N100N120N1 103NNO? ONO110NOOONONON21000ONNONNNNO01021112000NNNNO? 01301010120 NNONONO1 ON12ON220N11 33NN1 10NOPOON10ONONON21011 ONNONNNN001411014200NOONO2 313013301221 NO0212110010N240N1023NN100N? 110N? OONONON? 100 ? ONNONNNN0012121NNNNNNNNNO2 133120021121 NOO 1? 1411101 N12ON1 101NNO? ON12NNNNOONNN? N2ON401110NNNNO01521110201111202 01301021110 NNON1000NNNN1N120N1103NN120NO100N100NONON2ONO00001101100102? 102101200NO2 12200121110 NNONONO10N110N120N102????? ONO101N10ONONON2ON? OONNONNNNO0131111410?? 1120? 12302221101 NNOO2O21 0N10ON20120N212NNNONO101 NOOONON2? 2? 04? ONNONNNNO0100?????00NONNO2 223010121221 NOO 1 ? 0110020 N1 10N1 1 13NN21 P011 100N? OONONON212201000NNNNOO????????????? 12 14301012120 NNON? ?OONNNN1N1? ON1 103NN? ? ON1 2NNNNOONONON1 100? ONNONNNNO01211014200NOONO2 32301012110 NNON1? 110NO2ON33100N23NNO1111110NOOONONON212310NNONNNNO01021111112111100 O0201011120NNONONO1 ONP1ON23ON1 103NN1 10NO100N1 OONONON21000ONNONNNNO01112024? 00NOONO2 32201011120 NNON10010NO1 ON22120NO1? NNNON12NNNNOONONON? ON4? ONNONNNNOO1000NNNNNNNNNNO2 25101012220 NNON10010N12ON120N1 103NNO1 ONO11 PO0 111 ONONNONO011110P10001021102002113NO2 21301021220 NNON10010N120N120N1 103NN1 10NOONONOOONONONOONO011010110001021 102002112NO0 21301021220 NNON10010N12ON12ON1103NN110NO1120POONONONOONOO11011110001021112002110NOO 1 P20101P2120NO0 100111 POON220N1103NN010N1100N100NONON2ONOOPOOONNNNO010211201PONOONO2 1 P20101P2120NOO 100111 POON22ON1 103NNO10N110ON10ONONON21200POOONNNNO010211201PONOONO2 11301011210 NNONONNONNNN1N130N1103NN2100110ON? OONONON? 1200111 ONNNNOO102111 1100 NNNN12 21301022201 NNOO? ? OONNNN1 N23ON1OO3NN21 1002NNNNNONONON212301110NNNNO0 1021111102414N12 1530101 P1 1 ONN1 N1PO 1ONP2ON1 10N10112NNN1302NNNNOONON3N2ON3 1ONNONNNNO01021110002100NO2 31001012120 NNON1? O1ONO2ON120N1103NNO11310NON2OONONON212310NNONNNNO01021101112111100 $30201012110 N N O N 10110$ NO2ON110N1113NNO113110ONOOONONON212310NNONNNNOO1021111112111100 05301012110NNON2 1410N120N11 10N1 103NNO1ON1101NOOONONON111210NNONNNNOO1021100112011102 1530 $153010221121 N 30211110110 N 110 N 1113 N N 21101121$ N3OONONON21021ONNONNNNOO1021101120NNNN12

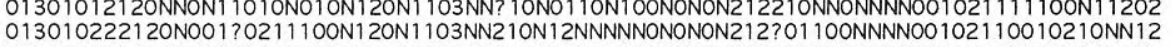
25312010111 NNOO2O22ONOO2123101P13NNO1ON1111NPOONONON211210NNONNNNO01021111112011102 25312010111 NNOO20220NO02123101P13NNO1ON1111NPOONONON211210NNONNNNO01021111112011102 $001012 \mathrm{P} 111$ ONNON1? 01 ONOOON220N1 103NN11 ONO 10ON30ONONON210010NNONNNNO01111002200NOONO2 242013301121 NO0212110010N120N1013NN1 10NO110N? OONONON1 1000ONNONNNN0012121 NNNNNNNNNO2 223010121221 N001? 0110020 N1 10N1 1 13NN21 1012NNNNNONONON210301000NNNNOO?????????????? 12 133010122221 NO0 100110020 N22ON1 103NN21100101 NOOONONON212201110NNNNO0 1021110102110 N12 1 P20101P2120NO0100111POON22ON1 103NNO1 ON1 10ON1 OONONON2ONO011010P1 10010211201PONOONO2 111010121221 NOO2 $10110020 N 110 N 1113 N N 21$ PO 1 POON? OONONON21220100ONNNNO01021110120NNNN12 100010121120 NOOONO110020N120N11011 NNNONO 111 NOOONONON2ONO01101101000ONNNNNNNNNNNNNO2 01301022220 NNON1000NNNN1N130N1103NNO10NO1021210N1320NONO01111211011?????????????0?

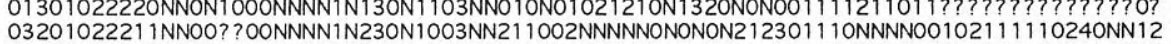
P330102012ONNON10120NO1ON22ON1 103NNO1132110N10ONONON21230ONNONNNNO01021111112111101 13101022120 NNON? ? 110 NO1 ON12ON1 1010NNNONOOO 1 NOOONONON2ONO01101101000ONNNNNNNNNNNNNO2 15111012111 NNOO20110NOO1N23101013NNO1131101N1OONONON212310NNONNNNO01021110112111102 11P10202120NO0120411101N230N1013NN211002NNNNNONONON212201000NNNND0102111110210NN12 O17P10202120NO 100010201121 NOO??0110010N221? 11011 NNNONO 101 N1OONONON2ONO01101211200ONNNNNNNNNNNNNO2 21101012110 NNON110ONNNN1N120N1103NNO1ON1100N10ONONON2ONO010111011001021110101200NO2 O2301012110NNON1?010NO2ON120N11010NNNON? 101 NOOONONON2ONO01101001000ONNNNNNNNNNNNNO2

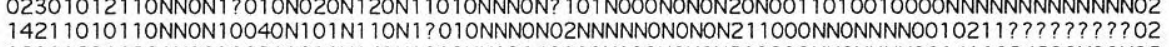
123012211221 NOO?? 2111000 N140N1013NN13140100N10ONONON2100?? NNONNNNO0141 1024200 NOONO2 102011101121 NOO301110010N130N1013NN11141121N10ONONON21000ONNONNNNO014121NNNNNNNNNO2

Tabla 2. Matriz de caracteres morfológicos (Tabla 1) distribuidos entre los géneros utilizados en los ensayos $[\mathrm{N}=$ no aplicable; $\mathrm{P}=$ polimórfico; ? = desconocido]. 
"favorable". En primer lugar, se utilizó una rutina de búsqueda heurística utilizando métodos de intercambio de ramas (global branch swapping) con incorporación aleatoria de los taxones y replicando el análisis diez veces. Los otros dos métodos de búsqueda que permite el programa (branch and bound searches y bootstrap replications) eran prohibitivamente lentos debido al tamaño y estructura de la matriz de datos.

\section{GRUPO EXTERNO}

Además de los resultados aquí presentados, de forma abreviada, se han realizado otros muchos análisis con los mismos taxones (grupo interno) pero distintos grupos externos. Así, por ejemplo, se utilizaron los más próximos morfológicamente a aquellos grupos en que se estaba más interesado; pero también otros no tan evidentemente relacionados, ya que aunque la sola presencia de uno o dos caracteres (e.g. braquióforos o crura largos, concha "lisa", plataformas para los músculos) pudiese inducir a suponer una conexión más o menos directa entre esas formas, quizás otros géneros, no tan próximos morfológicamente, pueden haber desarrollado dichas estructuras o características morfológicas muy rápidamente en una dirección particular. La ausencia, en muchos casos, de "intermedios" hace más difícil el estudio. Los cladogramas obtenidos, que sólo representan hipótesis de trabajo que necesitan ser puestas a prueba mediante nuevos análisis (y en el caso de aparecer datos nuevos), no difieren esencialmente de aquellos seleccionados para ser expuestos en este trabajo.

Como grupos externos se utilizaron: 1) géneros pertenecientes a la superfamilia Triplesioidea (e.g. Triplesia, Streptis, Plectotreta, Oxoplecia y Onychotreta), la más primitiva entre los "articulados" según aparece en los análisis realizados por Carlson (1991); 2) braquiópodos estróficos con un espiralio semejante al desarrollado en formas astróficas ("distróficas") pero que en otros aspectos, especialmente su mecanismo de charnela, recordaban órtidos y que aparecían en la base del clado que comprendía los atripoideos, atiridoideos, retzioideos, pentameroideos, rinconelloideos y terebratuloideos en los ensayos realizados por Carlson (1991, Fig. 4); 3) otros géneros, los primeros "articulados" y por tanto probablemente antepasados de todos los demás (e.g. Billingsella); 4) órtidos próximos morfológicamente a los braquiópodos con espiralio, como el estrófico Platystrophia o los "rostrados" Angusticardiniidae (Angusticardinia); 5) órtidos con braquióforos bien desarrollados, con las bases convergentes en un septo medio, como en Dalmanella, o posiblemente precursores de braquidios complicados (en forma de cinta o espiralados) como los presentes en Phragmorthis, en la ya mencionada Platystrophia o en formas con concha con puntos como Rhipidomella y Perditocardinia (forma rostrada, con interáreas inapreciables y línea de charnela muy estrecha); 6) pentaméridos como los sintrophiidinos, con la concha biconvexa y frecuentemente lisa, con reducción de las interáreas y de las líneas de charnela (típicas de los órtidos de los cuales probablemente

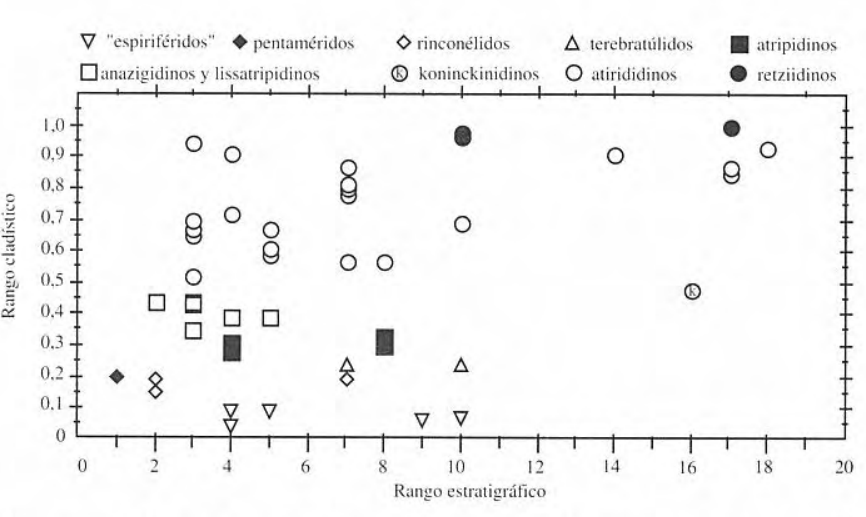

Figura 2. Representación del rango cladístico frente al rango estratigráfico (según Gauthier et al., 1988 y Carlson, 1993) de los taxones correspondientes al cladograma de la Fig. 1.

derivaron) dando lugar a conchas astróficas, rostradas, con pliegue medio dorsal e internamente con lamelas dentales, plataformas para el agarre de los músculos y procesos braquiales alargados comparables con crura (géneros utilizados: Porambonites, Camerella, Liricamera, Stenocamara, Plectocamara, Parastrophinella, Syntrophia, Xenelasma, y Brevicamera); 7) rinconélidos primitivos (como Ancistrorhyncha, Oligorhynchia, Drepanorhyncha y Dorytreta; Trigonirhynchiidae, como Rostricellula o Trigonirhynchia, y puntuados, como Rhynchopora) con concha biconvexa, uniplicada y con una forma muy sencilla de braquidio (que recuerda la de los pentaméridos, de los cuales probablemente hayan derivado neoténicamente por un desarrollo precoz de crura y la eliminación de cualquier tendencia a formar espondilio) pero externamente con ornamentación de costillas numerosas, e internamente, con dientes cirtomadontos bien desarrollados.

Observando los procesos (braquióforos o crura) desarrollados en algunos órtidos (e.g. Platystrophiinae, como Platystrophia), pentaméridos, como los porambonitoideos, o rinconélidos, parece razonable asumir que todos ellos compartieron la posibilidad o capacidad de desarrollar espiras.

Al realizar los análisis, en los que se utilizaron como grupos externos pentaméridos o rinconélidos, esos taxones (más los géneros que habían mostrado caracteres menos derivados en análisis previos) fueron excluidos de la matriz (grupo interno). En todos estos ensayos, los caracteres no fueron ni pesados ni ordenados.

\section{ANÁLISIS SELECCIONADOS}

Entre los múltiples análisis realizados se escogieron ocho como representativos del resto de ensayos (Figs. 114). En el primero, se incluyeron 55 géneros en el grupo interno y se utilizó un billingséllido y un dalmanéllido como grupo externo (Figs. 1 y 3). Ambos poseen concha de tamaño moderado, biconvexa, de convexidad débil a moderada, más pronunciada en la valva ventral, no rostrados, con el umbo ventral casi recto, con ornamentación radial y líneas de crecimiento suaves, 





espaciadas, no lamelosas; pliegue medio y seno ausentes o poco desarrollados; línea de charnela recta $(\approx$ a la máxima anchura de la concha); área cardinal ventral y dorsal extensas, con orientación apsa a ortoclina la ventral y anaclina la dorsal; abertura peduncular en forma de deltirio, abierto o cubierto completamente; carecen de soportes pedunculares y musculares (e.g. espondilio, plataforma cardinal, septalio, cruralio, etc.); dientes pequeños (deltidiodontos) o de tamaño medio; soporte para el lofóforo no mineralizado; primitivamente de concha impuntuada (billingséllidos), puntuada en los más derivados (dalmanéllidos). La mayor parte de los caracteres listados son considerados generalmente como típicamente primitivos (ver Williams y Wright, 1965 y Carlson, 1993).

En el segundo análisis, y con objeto de observar su efecto en los cladogramas previos, se añadió un retziidino más al grupo interno manteniéndose invariable el grupo externo (Fig. 4). En el tercer análisis, todos los taxones, excepto los terebratúlidos y los braquiópodos astróficos con espiralio, fueron eliminados de la matriz utilizada en el primer análisis, y un rinconélido primitivo, un cameréllido, y un protozygino fueron escogidos como grupo externo (Fig. 5).

En los siguientes análisis (IV-VIII), se puso atención especial en aquellos grupos principales de atíridos s.l. reconocidos en los análisis previos, y se utilizó como grupo externo dos rinconélidos primitivos (Ancistrorhyncha y Rostricellula) ya que habían aparecido constantemente en la base de los braquiópodos astróficos con espiralio, en los cladogramas resultantes en el primer análisis (Fig. 1). En el análisis se incluyeron 15 meristelloideos en el grupo interno (Fig. 7). En el V y VI, 26 atiridoideos fueron incluidos en la matriz (Figs. 9 y 10). Mientras que en el $\mathrm{V}$ todos los caracteres fueron igualmente pesados, en el VI un peso de 10 (base 1000) fue dado a dos caracteres básicos relacionados con el cardinalio, el tipo de plataforma cardinal y el grado de desarrollo del proceso cardinal (cardinal flanges). Para realizar los análisis VII y VIII, se incluyeron 19 retziidinos en la matriz (Figs. 12 y 14). En el análisis VII, todos los caracteres fueron igualmente pesados, sin embargo, para el VIII, un peso 10 (base 1000) fue asignado a dos caracteres relacionados con el aparato yugal (el tallo y las lamelas accesorias), con el fin de investigar su posible efecto en la clasificación de los retziidinos.

\section{COMPARACIÓN ENTRE LOS RANGOS CLADÍS- TICO Y ESTRATIGRÁFICO}

Información estratigráfica (datos de primera aparición de los taxones) y morfológica (rango cladístico, ver Gauthier et al., 1988 y Carlson, 1993) fueron combinadas y los resultados comparados con los diagramas obtenidos independientemente de cualquier posición estratigráfica, con la intención de comprobar si las informaciones obtenidas con ambos criterios de polaridad eran congruentes (Carlson, 1993).

\section{RESULTADOS}

\section{ANÁLISIS I - CLADOGRAMA 1}

El primer análisis produjo 186 cladogramas igualmente parsimoniosos, con una longitud de 732 pasos y un índice de consistencia de 0,294 (0,285 excluyendo los caracteres no informativos). Aunque el valor del índice de consistencia parece relativamente bajo, se mantiene dentro de la norma para análisis con 57 taxones (ver Sanderson y Donoghue, 1989; Klassen et al., 1991). Un cladograma en diagonal (slanted) (Fig. 1), con apomorfías definiendo nudos, puede servir como base para la discusión de los casos de homología y homoplasia en la evolución de los caracteres. Dada la edad (distribución estratigráfica) "cubierta" por los taxones de la matriz, que todos ellos (excepto los rinconélidos y los terebratúlidos) se han extinguido, y la relativa semejanza entre la mayor parte de los "braquiópodos articulados" (al menos, en comparación con, por ejemplo, artrópodos o vertebrados), se espera que la consistencia entre los caracteres sea relativamente baja, así como un nivel no muy alto de congruencia. El cladograma está enraizado en un nudo interno con politomía basal.

La concordancia entre la primera aparición estratigráfica de los grupos principales de braquiópodos con espiralio y otros grupos de "articulados", y su rango cladístico es bastante buena (Fig. 2), lo que sugiere que tanto los métodos tradicionalmente utilizados en paleontología (sistemática clásica), como los que utilizan grupos externos (sistemática filogenética) indican una dirección similar de polaridad de los caracteres en la evolución de los braquiópodos con braquidio espiralado. La mayor parte de los caracteres poseen un índice de consistencia inferior a 1,0 (0,294 de media), lo que indica la existencia de fenómenos de reversión, convergencia o paralelismo.

El cladograma de la Fig. 1 ordena diversos taxones como grupos hermanos [e.g. Eospirifer de "espiriféridos", puntuados o no; Ancistrorhyncha, de los rinconellidinos; Rostricellula, de Trigonirhynchia; Stenocamara, de Xenelasma; Cryptonella, de Dielasma; Zygospiraella, de la mayor parte de los atrípidos; Dayia, de Lissatrypa; Idiospira, de Manespira y Protozyga; Amphiclina, de Koninckina; Hyattidina, de la mayor parte de los meristelloideos y retzielloideos; Bifida, de Kayseria; Retziella, de Metathyrisina y Molongia; Cryptothyrella, del resto de meristelloideos; Athyris, de Pachyplax; Athyrisina, de Hexarhytis y Plicathyris; Pexidella, de Diplospirella (y Helenathyris); Eumetria, de Hustedia, Cassianospira y Neoretzia].

En el cladograma de la Fig. 1, se distinguen fácilmente once grupos que se corresponden con taxones ya existentes de rango superior al de subfamilia. "Espiriféridos" puntuados (espiriferínidos) e impuntuados (espiriféridos) forman un grupo bien definido en la base del cladograma, lejos de los braquiópodos astróficos con espiralio. Típicamente poseen conchas ventribiconvexas, moderadamente rostradas, puntuadas o impuntuadas, con pliegue y seno medios presentes en toda la valva, abertura peduncular en forma de deltirio (parcial o completamente 
cubierto), interárea cardinal dorsal reducida y ortoclina. El soporte mineralizado del lofóforo (un espiralio con los ápices dirigidos lateralmente, las lamelas primarias creciendo como prolongación directa de los crura, paralelamente al plano de simetría y muy próximas a él) en conjunción con las características externas previamente mencionadas, son diagnósticos para los "espiriféridos". Los resultados del análisis filogenético (Fig. 1) coinciden con esta caracterización general de los braquiópodos estróficos con espiralio.

Recientemente Rong y Zhan (1996) mostraron la presencia en Eospirifer praecursor, el eospiriférido más antiguo conocido, de un espiralio dirigido ventrolateralmente y con un par de pequeños "procesos yugales". Su relativa semejanza con el presente en algunos atripoides ordovícicos hace suponer a dichos autores que los eospiriféridos pudiesen haberse originado durante el Ordovícico superior a partir de un antecesor atripoide con espiralio muy sencillo dirigido ventralmente.

Los rinconélidos aparecen, junto con los pentaméridos, formando un clado bien definido en el cual Ancistrorhyncha y Dorytreta son grupos hermanos, en la base de los Trigonirhynchiidae los cuales se agrupan con los porambonitoideos (Pentamerida). Ambos grupos se caracterizan por ser astróficos, poseer pliegue medio y seno profundo (costado en la mayor parte de los rinconélidos), abertura peduncular en forma de deltirio que puede estar parcialmente cubierto (Trigonirhynchiidae). Los rinconélidos (excepto los estenoscismatoideos) carecen del espondilio característico de los pentaméridos (ver Carlson, 1993). Los rinconélidos primitivos presentan una plataforma cardinal disjunta; rinconélidos más recientes y pentameridinos poseen un cardinalio más elaborado (septalio los rinconélidos, plataforma dividida en tres partes los pentameridinos más derivados) frecuentemente soportado por un septo medio bien desarrollado. Tanto los rinconélidos como los pentaméridos carecen de proceso cardinal ya sea bilobado (típico de braquiópodos estróficos, deltidiodontos, paleozoicos) o estriado radialmente (el ctenophoridium de algunos "espiriféridos"). El lofóforo estuvo soportado por procesos braquiales, más o menos desarrollados, en los pentameridinos, y por crura en los rinconélidos. La estructura de la concha es impuntuada (sólo los rincoporoideos poseen concha con puntos). Ambos grupos, rinconélidos y pentaméridos muestran caracteres primitivos y derivados en varios estadios de transformación a partir de órtidos ancestrales.

Cryptonéllidos y dielasmátidos aparecen como grupos hermanos, lo que concuerda con su clasificación en el orden Terebratulida. Los terebratúlidos se caracterizan por poseer una concha astrófica, moderada a fuertemente rostrada, con el umbo ventral fuertemente curvado, foramen peduncular en posición meso a permesotírida y deltirio comúnmente cubierto por completo. Los cambios más importantes se producen en el cardinalio y en el braquidio. Cryptonéllidos y dielasmátidos poseen, respectivamente, una plataforma cardinal larga, perforada en su extremo posterior, profunda (a veces sésil) o un septalio amplio y descubierto. Un braquidio en forma de rizo o lazo aparece como soporte del lofóforo. La estructura de la concha es puntuada.

Los atripidinos forman otro grupo bien definido, morfológicamente intermedio entre terebratúlidos y lissatripidinos y grupos primitivos astróficos, con espiralio, los cuales se presentan en la Fig. 1 parafiléticamente junto con los koninckinoideos en la base de los grupos comúnmente clasificados como atíridos s.l.

Recientemente Boucot (en Boucot y Wilson, 1994) sugiere, tras el estudio de la ontogenia de terebratúlidos devónicos, su posible derivación a partir de atrípidos como Nalivkinia.

Los atrípidos aparecen al final del Ordovícico (Ashgill) desapareciendo al final del Devónico (Frasniense). Poseen conchas moderadamente rostradas, astróficas a casi estróficas, con la línea de charnela igual, o ligeramente menor que la máxima anchura de la concha. El exterior es costado, con lamelas de crecimiento bien desarrolladas, las cuales pueden extenderse anterolateralmente, enteras (frills) o formando espinas, típicamente alcanzan gran tamaño en los estadios adultos; el pliegue y seno medios son débiles, la valva dorsal es normalmente bastante más convexa que la ventral, siendo el perfil lateral frecuentemente convexoplano o incluso convexocóncavo. El área cardinal es reducida, el foramen peduncular, generalmente pequeño, se encuentra en posición meso a permesotírida en los taxones más primitivos y permeso a epitírida en los géneros más derivados; el deltirio se encuentra comúnmente cubierto parcialmente por placas deltidiales pequeñas (rara vez asociadas con collares pedunculares). Internamente, las lamelas dentales son generalmente cortas y convergen dorsalmente, las cavidades umbonales se encuentran en general pobremente definidas. En el davidsoniido Eifelatrypa, con caracteres derivados, el foramen se encuentra en posición submesotírida, el deltirio se encuentra completamente cubierto y carece de lamelas dentales. Como soporte mineralizado del lofóforo, los atrípidos poseen un espiralio con sus extremos dirigidos dorsalmente (dorsomedianamente en formas menos derivadas), lamelas primarias curvándose lateralmente desde los crura (en el plano comisural) y con procesos yugales. Este tipo de espiralio (común en todas las familias más "evolucionadas" de atrípidos), junto con los otros caracteres ya mencionados, es diagnóstico de los "atrípidos".

Los Dayiinae (Wenlock-Devónico Inferior), clasificados en el Treatise (Boucot et al., 1965, ver también Johnson, 1970) como atripidinos pero reasignados a los Athyrididina por Copper (1973a, b, 1986a, b), se agrupan constantemente, en este análisis (Figs. 1 y 3), con los lissatrípidos (Caradoc-Givetiense), entre formas con costillas (carácter primitivo) y lisas (derivado). Los lissatrípidos, como sugirió Copper (1973b), pueden ser una agrupación artificial ya que la condición lisa de la concha pudo haberse alcanzado, más o menos simultáneamente, a partir de formas costadas en diferentes líneas filogenéticas. Atrípidos con concha lisa 







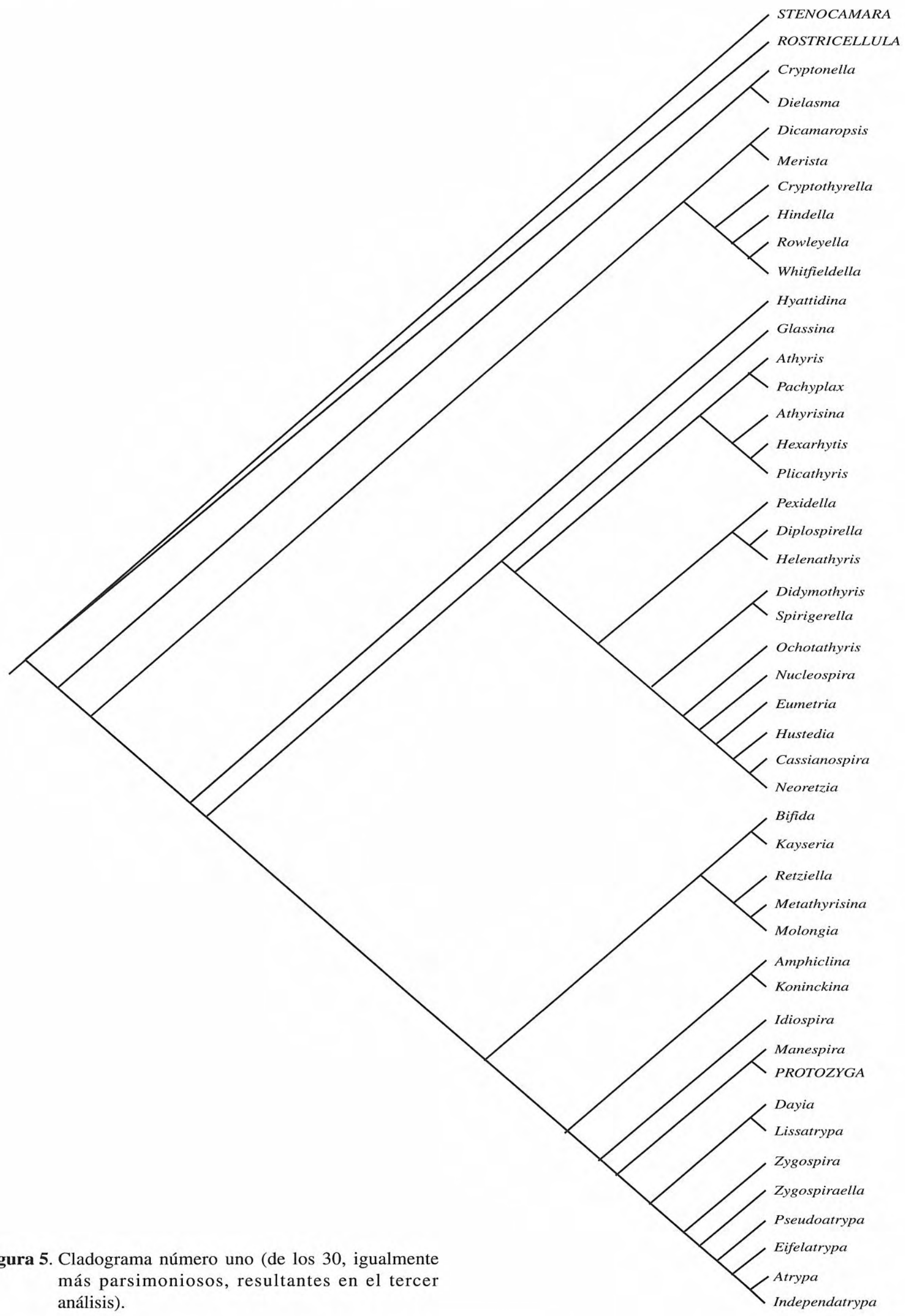


aparecen por primera vez en el Caradoc, pero Copper (1991) sugirió que su origen debió ser próximo al de los "atrípidos primitivos costados", es decir en el Llandeilo. Ambos grupos de atrípidos primitivos (anazigidinos y lissatripidinos), aunque surgiendo primero (Llandeilo?Caradoc) que los atripidinos (Ashgill) en el registro estratigráfico, parecen ser en este análisis morfológicamente más derivados que estos últimos. En los atrípidos, las lamelas primarias del espiralio se curvan lateralmente a partir de los crura, con sus extremidades dirigidas dorso o dorsomedianamente, menos frecuente medianamente. El yugo, en los casos en que se encuentra completo, se encuentra situado en la región anterior en las formas primitivas, desplazándose en dirección posterior en formas más modernas, pero situándose siempre en la región dorsal, bajo los conos espiralados.

Koninckina y Amphiclina son grupos hermanos, lo que concuerda con su clasificación en la familia Koninckinidae. Los koninckinoideos se caracterizan por poseer un perfil lateral plano o concavoconvexo, con la línea de charnela prácticamente estrófica; las extremidades apicales de los conos espiralados se dirigen en dirección ventral, poseyendo lamelas yugales accesorias, libres, intercaladas con los conos espiralados hasta sus ápices. Estas tres características, diagnóstico de los koninckinoideos, son muy diferentes de las que presentan el resto de grupos que poseen braquidio espiralado, lo que, junto con su distribución estratigráfica, hace difícil su clasificación ordinal (e.g. Boucot et al., 1965; Williams y Hurst, 1977; Grunt, 1989; Harper et al., 1993; Carlson, 1991; Dagis, 1996a).

El resto de braquiópodos astróficos con braquidio espiralado, es decir, todos los grupos frecuentemente clasificados como atíridos s.l., forman un grupo monofilético. El cambio más significativo se produce en el espiralio. La lamela primaria se curva posterodorsalmente a partir de los crura, paralelamente al plano de simetría; los ápices del espiralio se dirigen lateralmente; el yugo se encuentra siempre presente situándose ventralmente (entre los conos espiralados); las ramas yugales laterales surgen generalmente hacia la mitad de la longitud de la valva dorsal, se inclinan en dirección posterior en las formas primitivas mientras son verticales o se inclinan en dirección anterior en géneros más recientes; el arco yugal es agudo en algunas formas primitivas y redondeado en el resto; en formas avanzadas, el yugo se proyecta posterior, posteroventral o ventralmente en un tallo yugal y/o lamelas yugales accesorias; éstas pueden unirse con las ramas laterales del yugo o con el propio tallo yugal (formas primitivas) o permanecer libres y terminar posteriormente al punto de inicio de las ramas yugales laterales, o prolongarse entre los conos espiralados en formas más modernas. Raramente las lamelas yugales accesorias se unen a la mitad ventral de la lamela primaria del espiralio (Neoretziinae).

Hyattidina aparece en la base de dos grupos hermanos, los retziéllidos (más Bifida y Kayseria) y los meristelloideos; y Glassina, en la base de los atiridoideos, nucleospiroideos y retziidinos. Nucleospira aparece en la base de los retziidinos, los cuales forman también un grupo monofilético, el más derivado de todos los "articulados" de la matriz.

Existe una correlación positiva entre la primera aparición de los distintos taxones y el rango cladístico $(r=0,44)$ lo que indica la congruencia existente entre ambos criterios de polaridad (Fig. 2). Cuando se considera sólo el clado de los atíridos, (Fig. 1, nudo AA) el valor de este coeficiente se eleva hasta 0,61 .

Una hipótesis de trabajo, explícita y comprobable, de las relaciones genealógicas entre los braquiópodos astróficos con espiralio, frecuentemente clasificados como atíridos s.l., se muestra en los cladogramas obtenidos en los análisis III a VIII del presente trabajo (Figs. 5, 7, 9, 10, 12 y 14).

\section{CONSENSO DE LOS RESULTADOS OBTENIDOS EN EL ANÁLISIS I}

Aunque un árbol de consenso no constituye una base idónea para establecer una hipótesis filogenética, sí indica aquellas partes del árbol en las que las uniones permanecen constantes a la vez que proporciona una estimación del soporte que tienen las ramas conflictivas. El cladograma consenso (50\%) de los 186 cladogramas igualmente parsimoniosos obtenidos al realizar el análisis I se ilustra en la Fig. 3. Sólo 12 nudos no se encuentran soportados por el $100 \%$ de los cladogramas (4 por el $97 \%, 2$ por el $81 \%, 1$ por el $66 \%, 1$ por el $61 \%, 2$ por el $58 \%$ y 2 por el $57 \%$ ). La forma del diagrama consenso es prácticamente idéntica a la del cladograma primero (comparar Figs. 1 y 3). El clado con los "atrípidos primitivos costados" colapsa en una politomía y Ochotathyris se agrupa con Didymothyris y Spirigerella.

\section{ANÁLISIS II}

Este análisis constituye un buen ejemplo de cómo la inclusión, o la supresión, en la matriz, de sólo un taxón, puede tener una gran influencia en los resultados. Como ensayo, se incluyó en la matriz un quinto retziidino, Rhynchospirina. El número de cladogramas obtenido aumentó espectacularmente, de 186 a 612, cada uno con una longitud 752 y un índice de consistencia de 0,287. La topología de las relaciones entre los grupos principales permanece estable, tal como se puede ver en el cladograma consenso (50\%) de la Fig. 4. Es evidente, sin embargo, una cierta reorganización entre los grupos de braquiópodos astróficos con espiralio, no atrípidos.

Los atiridoideos se movieron hasta la base del grupo, a la vez que se aumentaba su resolución. Glassina y Hyattidina aparecen ahora juntos, parafiléticamente respecto a todos los demás atiridoideos los cuales se presentan ahora monofiléticos con Athyris en la base de todos ellos. Nucleospira se desplaza a una posición entre los clados con los atiridoideos y los meristelloideos. Los meristelloideos se presentan como grupo hermano de retziidinos y retzielloideos. En este cladograma consenso, Bifida y Kayseria se trasladan a una rama entre los retziidinos y los retzielloideos, como grupo hermano de estos últimos. Por tanto, la inclusión en la matriz de sólo un taxón más, Rhynchospirina, desplaza significativa- 


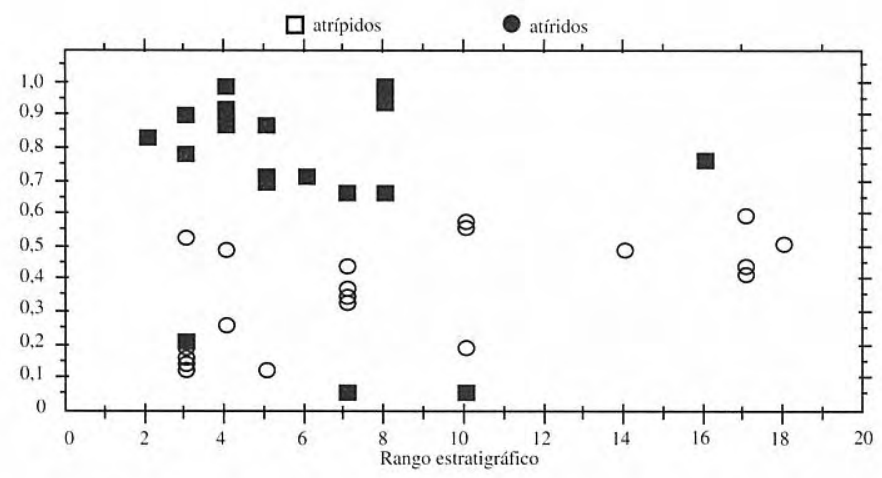

Figura 6. Representación del rango cladístico frente al rango estratigráfico.

mente las afinidades filogenéticas entre los braquiópodos astróficos, con braquidio espiralado, no atrípidos.

La correlación entre los datos de primera aparición, con el rango cladístico de los taxones, resultante del análisis, proporciona un coeficiente de correlación mucho menor $(r=0,27)$. El valor de este coeficiente disminuye considerablemente en el caso de que consideremos sólo el clado con los atíridos s.l. $(\mathrm{r}=-0,07)$ mostrando una falta de congruencia entre la hipótesis filogenética representada en la Fig. 4 y los datos estratigráficos, y por tanto indicando que las afinidades filogenéticas se encuentran mejor representadas por la hipótesis representada en la Fig. 1.

\section{ANÁLISIS III - CLADOGRAMA 1}

En el tercer análisis, todos los taxones, excepto los terebratúlidos y los braquiópodos astróficos con espiralio, fueron eliminados de la matriz utilizada en los análisis previos. Como grupo externo se utilizaron un rinconélido primitivo, un cameréllido y un protozygino, ampliamente considerados como ancestros de los braquiópodos astróficos con espiralio (ver también la Fig. 1), aparte de poseer representantes más antiguos geológicamente. El análisis produjo 30 cladogramas igualmente parsimoniosos, de longitud 609 y un índice de consistencia de 0,337 (Fig. 5). Los meristelloideos forman un clado cerca de la base del cladograma, justo por encima de los terebratúlidos. Hyattidina aparece en la base de los atiridoideos, retziidinos, retzielloideos y atrípidos, los cuales también forman clados. Glassina, como ocurría en el primer análisis, aparece en la base de los atiridoideos, nucleospiroideos y retziidinos. Bifida y Kayseria, que aparecían como grupo hermano con los retzielloideos (y ambos de los meristelloideos) en el análisis I, se desplazan, en este análisis, hasta un lugar entre atiridoideos y atripoideos (comparar las figuras $1 \mathrm{y}$ $5)$. Los koninckinoideos se muestran como grupo hermano de los atrípidos s.l. El grupo interno no es monofilético, Protozyga comparte más antepasados comunes con los atrípidos que con otros braquiópodos astróficos con espiralio. Protozyga se agrupa con otros atrípidos primitivos de edad similar, en la base de los lissatrípidos (silúricos) y los atrípidos devónicos, las formas más derivadas dentro del clado de los atrípidos. El diagrama consenso muestra una morfología semejante a la del cladograma primero a pesar de que Idiospira, en lugar de ser parafilético, forma un grupo hermano con el clado formado por Protozyga y Manespira.

No hay correlación entre los datos de primeras apariciones y el rango cladístico resultante del análisis $(\mathrm{r}=0,05)$. Cuando se consideran sólo los atiridoideos, meristelloideos y retziidinos, el valor del coeficiente de correlación aumenta $(\mathrm{r}=0,63)$, indicando que, al menos dentro de este clado, existe congruencia entre la hipótesis filogenética representada en la Fig. 5 y los datos estratigráficos (Fig. 6).

\section{ANÁLISIS IV - CLADOGRAMA 1}

Todos los taxones, excepto los meristelloideos, fueron eliminados de la matriz utilizada en los análisis previos. De esta forma fue posible incrementar el número de meristelloideos hasta un total de 15 géneros (ver la Fig. 7), e investigar en detalle sus relaciones filogenéticas. Como grupo externo se utilizaron rinconélidos primitivos, considerados como antepasados de los braquiópodos astróficos con espiralio (ver la Fig. 1).

Se obtuvieron 3 cladogramas igualmente más parsimoniosos de longitud 168 y un índice de consistencia de 0,649 (0,617 si se excluyen aquellos caracteres no informativos). La concordancia entre las primeras apariciones en el registro estratigráfico y su rango cladístico es muy buena ( $r=0,71$; Fig. 8). Cuatro grupos de géneros son fácilmente reconocibles. Meristéllidos primitivos forman un clado en la base del cladograma, siendo Hindella grupo hermano de Koigia y Whitfieldella. Cryptothyrella y Meristina aparecen parafiléticamente en la base del clado con el resto de meristellinos. El nudo I corresponde al punto en el cual los merístidos son generalmente reconocidos hoy día. Camarospira aparece en la base de los camarophorellinos y rowleyellinos y Dicamaropsis de los meristinos. El diagrama consenso (todos los nudos se encuentran soportados por el cien por cien de los cladogramas) muestra la misma morfología que la del cladograma primero si bien Dicamaropsis y Camarium forman un grupo hermano en lugar de ser parafiléticos ("peine de Hennig").

\section{ANÁLISIS V - CLADOGRAMA CONSENSO (50\%)}

En este análisis, se incluyeron 26 atiridoideos en la matriz y se emplearon, como grupo externo, los mismos rinconélidos primitivos utilizados en los análisis previos. Realizado el análisis, se obtuvieron dos cladogramas de longitud 307 y un índice de consistencia de 0,472 (0,443 excluyendo los caracteres no informativos).

En el cladograma consenso (50\%) todos los nudos se encuentran soportados por el $100 \%$ de los cladogramas (Fig. 9). En la base, aparecen dos géneros primitivos junto con un atiridoideo triásico peculiar, Pexidella. La mayor parte de los géneros "lisos" aparecen, en gran politomía, entre esos atiridoideos primitivos y aquellos con ornamentación radial de costillas o pliegues, los cuales se agrupan en el extremo distal del cladograma. 

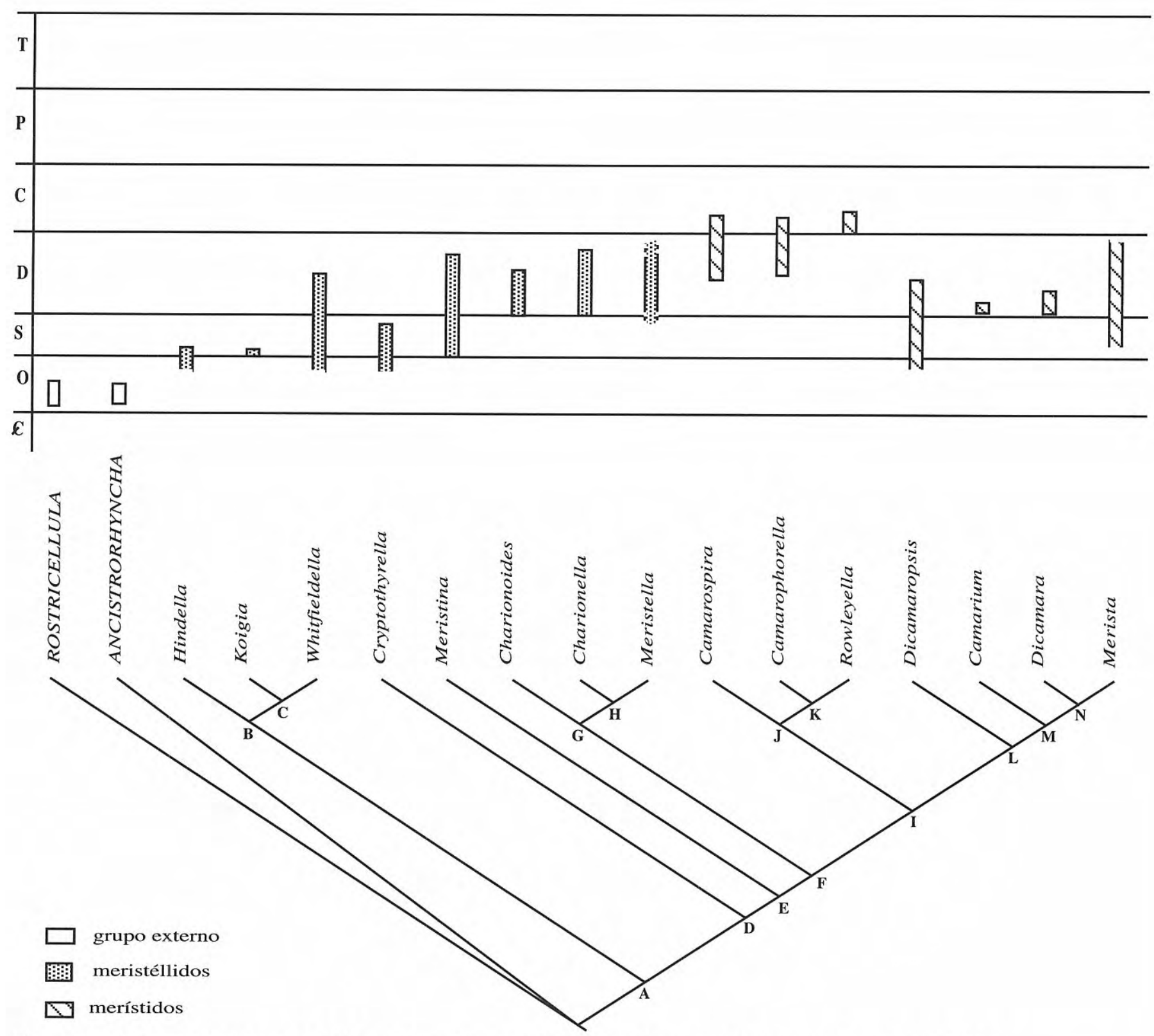

Figura 7. Cladograma número uno (de los 3, igualmente más parsimoniosos, resultantes en el cuarto análisis).

\section{ANÁLISIS VI - CLADOGRAMA 5}

En este análisis, la matriz (grupos interno y externo) era idéntica a la utilizada para realizar el análisis $\mathrm{V}$, si bien se dio un peso 10 a dos caracteres relacionados con el cardinalio, el tipo de plataforma cardinal y el grado de desarrollo del proceso cardinal (cardinal flanges). En el análisis se obtuvieron 7 cladogramas igualmente más parsimoniosos de longitud 383 y con un índice de consistencia de $0,520(0,499$ excluyendo los caracteres no informativos). Las relaciones así obtenidas (Fig. 10) se aproximan más a las «filogenias tradicionales» que las surgidas en los análisis previos (comparar Figs. 9 y 10). En la base del cladograma están los atíridos primitivos con concha claramente astrófica, tamaño pequeño a moderado, sin ornamentación radial, líneas de crecimiento débilmente marcadas (no lamelosas), con la valva ventral comúnmente más convexa que la dorsal, pliegue medio y seno poco desarrollados, plataforma cardinal perforada en el ápice, proceso cardinal ausente o rudimentario, septo medio dorsal, o miofragma, generalmente ausentes, visera y tallo yugales ausentes o presentes, comúnmente con las lamelas yugales accesorias terminando en las proximidades de las ramas yugales laterales.

Collarothyris y Didymothyris son taxones hermanos. Desarrollan soportes pedunculares (carácter derivado) pero comparten con Xenosaria un carácter primitivo, la posesión de sólo deltirio en lugar de deltirio más foramen, como tienen el resto de atiridoideos. En este análisis, Helenathyris no se une con Pexidella y Diplospirella (comparar e.g. Figs. 1 y 10), dos taxones triásicos que comparten con Helenathyris un carácter derivado, la posesión de lamelas accesorias yugales largas intercaladas con los conos espiralados hasta sus ápices. Helenathyris aparece en la base de tres pequeños clados de atiridoideos devónicos que poseen conchas 
biconvexas, de tamaño moderado, que pueden estar más o menos plegadas, generalmente con líneas de crecimiento muy juntas y lamelosas (a veces terminando en espinas sólidas), pliegue medio y seno bien desarrollados, plegamiento adulto generalmente alterno pero los plicatiridinos desarrollan un plegamiento mixto característico (Álvarez, 1990), astróficos a casi estróficos (e.g. algunos plicatiridinos), con lamelas dentales claras pero careciendo de soportes para el pedúnculo. Los atirisininos, que habían sido clasificados como una superfamilia de los retziidinos (Boucot et al., 1965), se agrupan, en los análisis mostrados en el presente trabajo (Figs. 1, 3, 4, 5, 6, 9 y 10), constantemente con los atíridos entre los atiridoideos y los plicatiridinos, en la base de éstos últimos (ver también Likharev et al., 1960; Rong et al., 1994). El análisis muestra también que Cleiothyridina, y Cryptospirifer están estrechamente relacionados con atiridinos no costados, tales como Athyris y Pachyplax, que aparecen como taxones hermanos. Composita y Spirigerella, y Diplospirella y Pexidella, aparecen como taxones hermanos en la base de los diplospiréllidos triásicos más derivados.

El nudo R puede servir para delimitar los atíridos con caracteres derivados característicos tales como la posesión de una plataforma cardinal no perforada y proceso cardinal (cardinal flanges) muy desarrollado. En el cladograma consenso sólo tres nudos no se encuentra soportados por el $100 \%$ de los cladogramas (el nudo O por $57 \%$ y los nudos $\mathrm{P}$ y Q por el 86 por ciento). La morfología del cladograma consenso es prácticamente igual a la del cladograma 5 (Fig. 10), con Cryptospirifer formando un clado (pobremente soportado) con Athyris y Pachyplax, y la unión con Ochotathyris y Oxycolpella colapsa en una dicotomía.

La comparación de los datos de primera aparición con el rango cladístico, obtenido en este análisis, indica una correlación muy buena entre ambas variables $(r=0,82$, Fig. 11), y soporta por tanto el esquema filogenético indicado.

\section{ANÁLISIS VII - CLADOGRAMA 1}

Para realizar este análisis se colocaron 19 retziidinos en la matriz y los mismos rinconélidos primitivos como grupo externo. Se obtuvieron tres cladogramas de longitud 199 y un índice de consistencia de 0,558 (0,522 excluyendo los caracteres no informativos). La mayor parte de los caracteres tienen un índice de consistencia menor de 1,0 (0,558 de media), indicando la existencia de diversos casos de inversión, convergencia o paralelismo. El acuerdo entre la primera aparición estratigráfica de los principales grupos de retziidinos y su rango cladístico es muy bueno, sugiriendo que ambos métodos, el tradicional (e.g. Boucot et al., 1965; Dagis, 1974; Grunt, 1986) o utilizando grupos externos (Fig. 12) indican direcciones de polaridad en los caracteres muy semejantes, en la evolución de los retziidinos ( $\mathrm{r}=0,86$, Fig. 13). Tres grupos de géneros son fácilmente reconocibles. Los mongolospiroideos aparecen en la base del cladograma. Poseen conchas astróficas, puntuadas y lisas, con palintropo moderadamente desarrollado, lamelas dentales

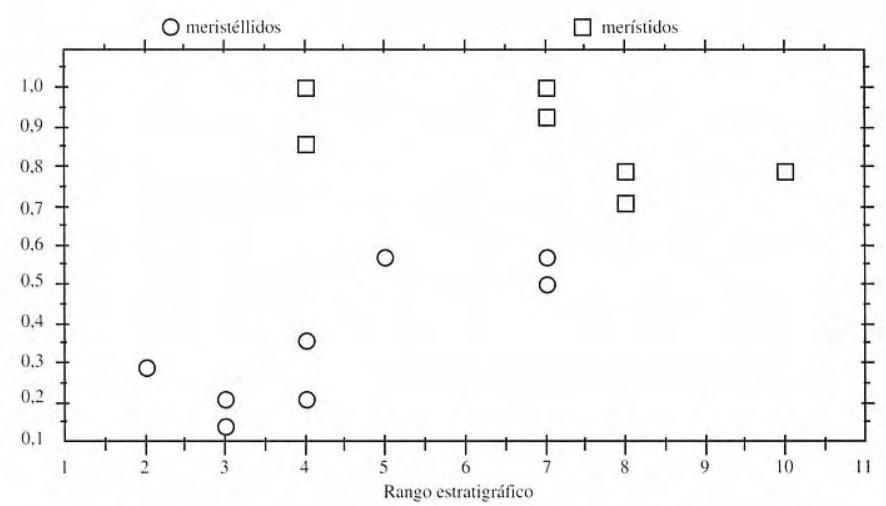

Figura 8. Representación del rango cladístico frente al rango estratigráfico.

y collar peduncular muy cortos, carecen de caracteres derivados como las lamelas de crecimiento y el corto proceso yugal que en forma de tejado y dirigido hacia atrás se encuentra presente en algunos rincospirinoideos y el tallo y las lamelas accesorias yugales de retzioideos más modernos.

Los rincospirinoideos comprenden un clado, bien resuelto, caracterizado por la posesión de una plataforma cardinal con las placas cardinales internas poco, o nada, desarrolladas. Espinas huecas, largas, numerosas dirigidas anterior o anterolateralmente caracterizan los parazígidos que aparece en la base de todos los demás rincospirinoideos. Homoeospira y Homoeospirella son géneros hermanos y parece que posean los caracteres más derivados en el grupo, a pesar de su aparición, ligeramente más temprana, en el registro estratigráfico. El nudo I puede servir para delimitar los retzioideos; siete apomorfías caracterizan el nudo, muchas de las cuales son características típicas de los retzioideos, tales como la plataforma cardinal no perforada con proceso cardinal (cardinal flanges) moderado a muy desarrollado, yugo careciendo de cualquier tipo de visera pero desarrollando un tallo largo y, en los géneros más recientes, lamelas yugales accesorias más o menos elaboradas. Retzia, Eumetria y Acambona constantemente se agrupan en la base del clado. Poseen tamaño grande, son astróficas y costadas, con plegamiento alterno suave y en el interior, generalmente con lamelas dentales como los rincospirinoideos, pero con una plataforma cardinal no perforada y un tallo yugal semejante al de los neoretziidos. Los últimos neoretziidos poseen una concha casi estrófica, con el umbo ventral casi recto e internamente con las lamelas yugales accesorias largas, que se intercalan con los conos espiralados principales hasta su ápice o que, en ocasiones, conectan secundariamente con la mitad ventral de la primera lamela del espiralio.

\section{ANÁLISIS VIII}

Para este experimento se pesaron dos caracteres (77 y 80), diez veces más que los otros 81 restantes. Se escogieron esos caracteres (relacionados con la presencia de tallo yugal y el desarrollo de las lamelas yugales accesorias) en particular, debido a que en estudios 


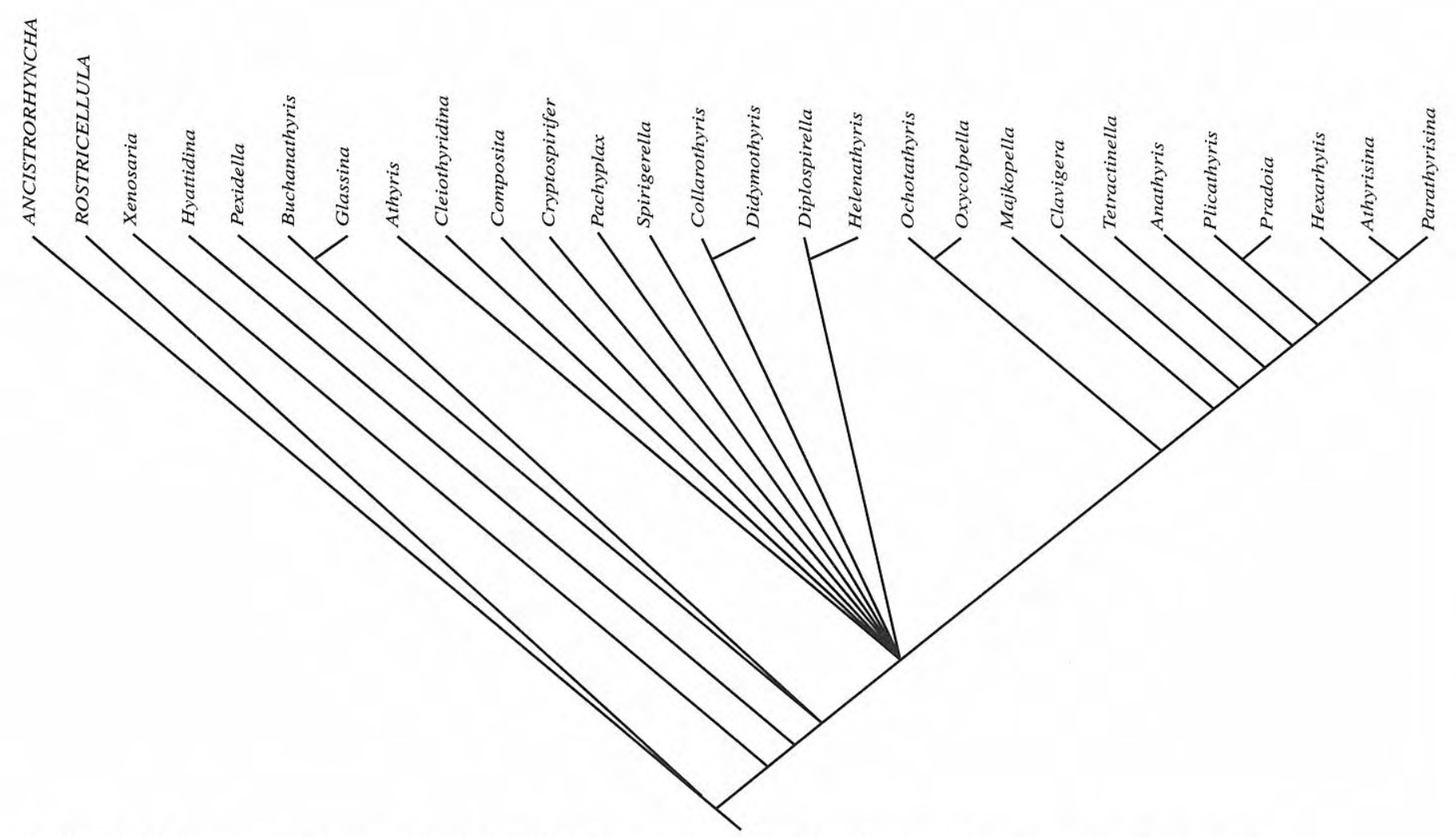

Figura 9. Cladograma consenso $(50 \%)$ de los 2 cladogramas más parsimoniosos resultantes en el quinto análisis.

previos de la filogenia de los retziidinos, diversos autores (e.g. Dagis, 1974; Alekseeva, 1977; Grunt, 1986) habían resaltado la importancia que en ellos había tenido el estudio del desarrollo del yugo. El análisis dio un solo árbol más parsimonioso, de longitud 255 e índice de consistencia de $0,647(0,625$ excluyendo los caracteres no informativos) siendo el resultado (Fig. 14) prácticamente idéntico al anterior (Fig. 12). En este análisis, Retzia, Eumetria y Acambona en lugar de aparecer parafiléticamente en la base del clado, se agrupan. Otros dos grupos hermanos, observados en análisis previos se mantienen en esta morfología (Hustedia de Thedusia; y Cassianospira de Hungarispira).

\section{DISCUSIÓN}

Las hipótesis filogenéticas obtenidas con estos análisis pueden servir como base para el entendimiento de las tendencias evolutivas desarrolladas entre los braquiópodos con braquidio espiralado, y en especial, entre aquellos generalmente considerados como atíridos s.l. Ha de tenerse en cuenta que en las transformaciones temporales, caracteres considerados como derivados con respecto a un nudo cerca de la base, pueden ser primitivos respecto a otros nudos en la zona superior de los cladogramas (cf. Carlson, 1992, 1993).

Línea de charnela. La línea de charnela es ancha y recta (carácter primitivo) en el grupo externo y en los espiriféridos s.l. Se acorta, volviéndose astrófica en el resto de "articulados" (más derivados). Pero existen algunas reversiones (e.g. diplospiréllidos). Líneas de charnela casi rectas se presentan en distintos grupos de braquiópodos con braquidio espiralado: atrípidos, koninckinidinos, neoretziidos, plicatiridinos y nucleospiroideos entre otros. Como ocurre en los sintrophiidinos (cf. Carlson, 1993), la condición más o menos estrófica (o astrófica) puede variar considerablemente incluso dentro de un género o una especie (e.g. Álvarez, 1990).

Abertura peduncular. Rinconélidos, algunos órtidos y espiriféridos s.l. poseen foramen en posición subapical. Los terebratúlidos y la mayor parte de los braquiópodos astróficos con espiralio (atirididinos y retziidinos) se caracterizan por poseer un foramen transapical. El pedúnculo, que emerge inicialmente en una posición subapical, emigra a través del ápice ventral (desde una posición meso a otra epitírida), gracias a la habilidad del epitelio para resorber calcita, proporcionando al braquiópodo algunas ventajas funcionales (ver, por ejemplo, Rudwick, 1970). En ambos casos, la abertura deltirial en su totalidad o en parte no es necesaria para el pedúnculo, siendo entonces obturada con placas deltidiales (algunos rinconélidos, atíridos y la mayor parte de los atrípidos) o por un deltidio o simfitio (e.g. algunos diplospirellidos, neoretziidos y terebratúlidos).

Tamaño y volumen. La convexidad de las valvas adultas permanece moderada en los rinconélidos, pero se vuelve fuerte en algunos "espiriféridos", pentameridinos, atrípidos (especialmente en géneros devónicos, derivados), retziidinos, atiridoideos y meristéllidos. El 



Figura 10. Cladograma número cinco (uno de los 7, igualmente más parsimoniosos, resultantes en el sexto análisis).

tamaño general también aumenta considerablemente a lo largo de todos esos linajes. En general se admite que en los braquiópodos, la capacidad de filtrado es función del área ocupada por los filamentos del lofóforo y por tanto, de la superficie total de los conos del braquidio. Influidos, hasta cierto punto, por su estrategia para asegurar su estabilidad, los atíridos siguieron dos líneas para aumentar el volumen de los conos espiralados, y teniendo el mismos efecto, un incremento en la superficie, y por tanto en la eficiencia metabólica del lofóforo; $\boldsymbol{a}$, aumento en el diámetro basal de los conos; $\boldsymbol{b}$, aumento en la altura del cono, asociado con un incremento en la anchura de la concha. Atiridoideos y meristéllidos constituyen dos buenos ejemplos de formas que desarrollaron la primera estrategia. En ellos, la eficacia del lofóforo se veía aumentada mediante el desarrollo de pliegue y seno medios facilitando la separación y canalización de las corrientes de entrada y salida. En los plicatiridinos, la eficiencia del lofóforo se vio acrecentada al aumentar su longitud, gracias al sucesivo incremento en longitud de la concha. Este desarrollo junto con la adquisición de un plegamiento mixto (Álvarez, 1990), facilitó la separación de las corrientes. Otros atíridos siguieron una estrategia intermedia. Aumentaron el área basal de los conos espiralados mediante el desarrollo de pliegues en ambas valvas a la vez que crecían en anchura. Aunque un tamaño grande y una convexidad adulta fuerte parecen caracteres derivados, algunos merístidos, neoretziidos y atíridos triásicos poseen una concha pequeña de moderada convexidad.

Dentición. Los braquiópodos con dentición cirtomatodonta no parecen constituir un grupo estrictamente monofilético (ver Jaanusson, 1971; Carlson, 1989, 1992, 1993; Williams et al., 1996). Los rinconélidos, terebratúlidos, braquiópodos astróficos con braquidio espiralado (e.g. Álvarez y Brunton, 1990a) y algunos pentaméridos poseen dientes cirtomatodontos de tamaño moderado a grande [carácter más derivado que la presencia de pequeños dientes (dentición deltidiodonta) que no permiten una trabazón fuerte]. 
Plataformas musculares. En la región posterior de ambas valvas a veces se desarrollan diversos tipos de plataformas. Sirven para elevar, y en algunos casos para proyectar posteriormente, el lugar de agarre de los músculos por encima de la superficie interna de las valvas (ver Rudwick, 1970; Carlson, 1993; Brunton et $a l ., 1996)$. En la valva ventral, el espondilio (ausente en los rinconélidos, excepto en los estenoscismatoideos) es diagnóstico de los pentaméridos (ver Carlson, 1993). Una plataforma arqueada, comúnmente con una cámara debajo, unida posterior y lateralmente al interior de la valva, y libre y bastante elevada en su parte media que puede soportar la lamelas dentales, es característica de los merístidos (shoe-lifter process de King, 1846, definido por Williams y Rowell, 1965, p. H152). Unos pocos espirigerellinos poseen también espondilio. En la valva dorsal, los rinconélidos primitivos tienen una plataforma cardinal disjunta, rinconélidos más recientes poseen septalio, y los pentameridinos un cardinalio más elaborado, poseyendo, los más derivados, una plataforma dividida en tres partes. En los terebratúlidos se produjeron cambios importantes en el cardinalio. Plataformas perforadas apicalmente son características de los cryptonéllidos, mientras que septalios, largos, profundos (a veces sésiles), anchos y descubiertos aparecen en los dielasmátidos. Diferentes tipos de plataformas cardinales disjuntas aparecen también en atrípidos, hyatidínidos y rincospirinoideos. La mayor parte de los atiridoideos poseen una plataforma perforada en su ápice, mientras que retzioideos, diplospiréllidos y algunos retzielloideos desarrollan una plataforma más derivada, no perforada. En terebratéllidos actuales y en muchos géneros extinguidos, las placas cardinales internas, soportando los músculos adjustores dorsales, forman una especie de artesa soportada generalmente por el septo medio dorsal. Esta estructura (septalio), es característica de la mayor parte de los meristelloideos. Menos frecuentemente, las placas cardinales internas se unen directamente a la superficie interna de la valva, formando una estructura en forma de $\mathrm{V}$ en sección transversa. El septalio de algunos rinconélidos primitivos se encuentra completamente cubierto por placas (cover plates o connectivum) (Havlícek, 1961; Westbroek, 1967, 1969; Brunton et al., 1996), la mayor parte de los retzielloideos y algunos Trigonirhynchiidae poseen un septalio parcialmente cubierto (ver Rong et al., 1987, 1994; Jin et al., 1993; Brunton et al., 1996). De acuerdo con los resultados de los análisis I y II, entre los "braquiópodos articulados" se desarrolló, independientemente, un septalio al menos en cinco ocasiones.

Un proceso cardinal bilobado, característico de géneros estróficos deltidiodontos, no se desarrolla ni en los braquiópodos estróficos con espiralio (éstos poseen frecuentemente un proceso estriado radialmente, en forma de peine, llamado ctenoforidio) ni en todos los grupos astróficos. Rebordes cardinales posteriores (cardinal flanges) más o menos desarrollados son característicos de atirididinos y neoretziidos. Los músculos diductores que inicialmente se sujetaban directamente al gancho dorsal a través del foramen dorsal



Figura 11. Representación del rango cladístico frente al rango estratigráfico

(de la plataforma cardinal), se desplazaron, en especies del Paleozoico superior, al margen posterolateral de la plataforma cardinal, la cual se fue engrosando, proyectando ventralmente y frecuentemente bifurcando en dirección posteroventral (ver Álvarez, 1990; Álvarez y Brunton 1990a, b; Brunton et al., 1996).

La mayor parte de los merístidos poseen una plataforma arqueada (shoe-lifter) en la valva dorsal. Recientemente, Álvarez y Brunton (1995) erigieron una subfamilia, Rowleyellinae, con aquellos merístidos caracterizados por la presencia de un shoe-lifter dorsal y un cruralium, que se continúa anteriormente más que el shoe-lifter. Los pentaméridos poseen plataformas semejantes, asociadas o no a un septo medio, e interpretadas como asiento de músculos aductores. De acuerdo con los resultados de los análisis I, II y III, un cruralio ha aparecido independientemente en los merístidos y en 6 grupos diferentes de pentaméridos (ver Carlson, 1993).

Rudwick (1970) y Carlson (1993) discutieron ampliamente las posibles ventajas funcionales adquiridas por los taxones que desarrollaron alguno de estos tipos de plataformas musculares.

Braquidio. En los pentaméridos, el lofóforo fue soportado por procesos braquiales, más o menos largos, y en rinconélidos por crura. Estos procesos, las formas más simples de braquidio, pueden haberse originado independientemente en ambos órdenes (Rudwick, 1970). En los terebratúlidos, además de producirse importantes modificaciones en el cardinalio, se mineralizó un soporte en forma de rizo o lazo para el lofóforo, soporte característico del orden. Los espiriféridos (sensu Boucot et al., 1965) desarrollaron braquidios espiralados más o menos complejos. Durante el crecimiento de estos braquidios derivados, el epitelio que lo reviste desarrolla la posibilidad de resorber calcita y por tanto de crecer libremente formando estructuras muy complicadas.

Los "espiriféridos estróficos", puntuados e impuntuados, que forman un clado en la base del cladograma resultante en los análisis filogenéticos (Figs. 1, 3 y 4), desarrollaron un espiralio con los ápices dirigidos lateralmente y las lamelas primarias creciendo 


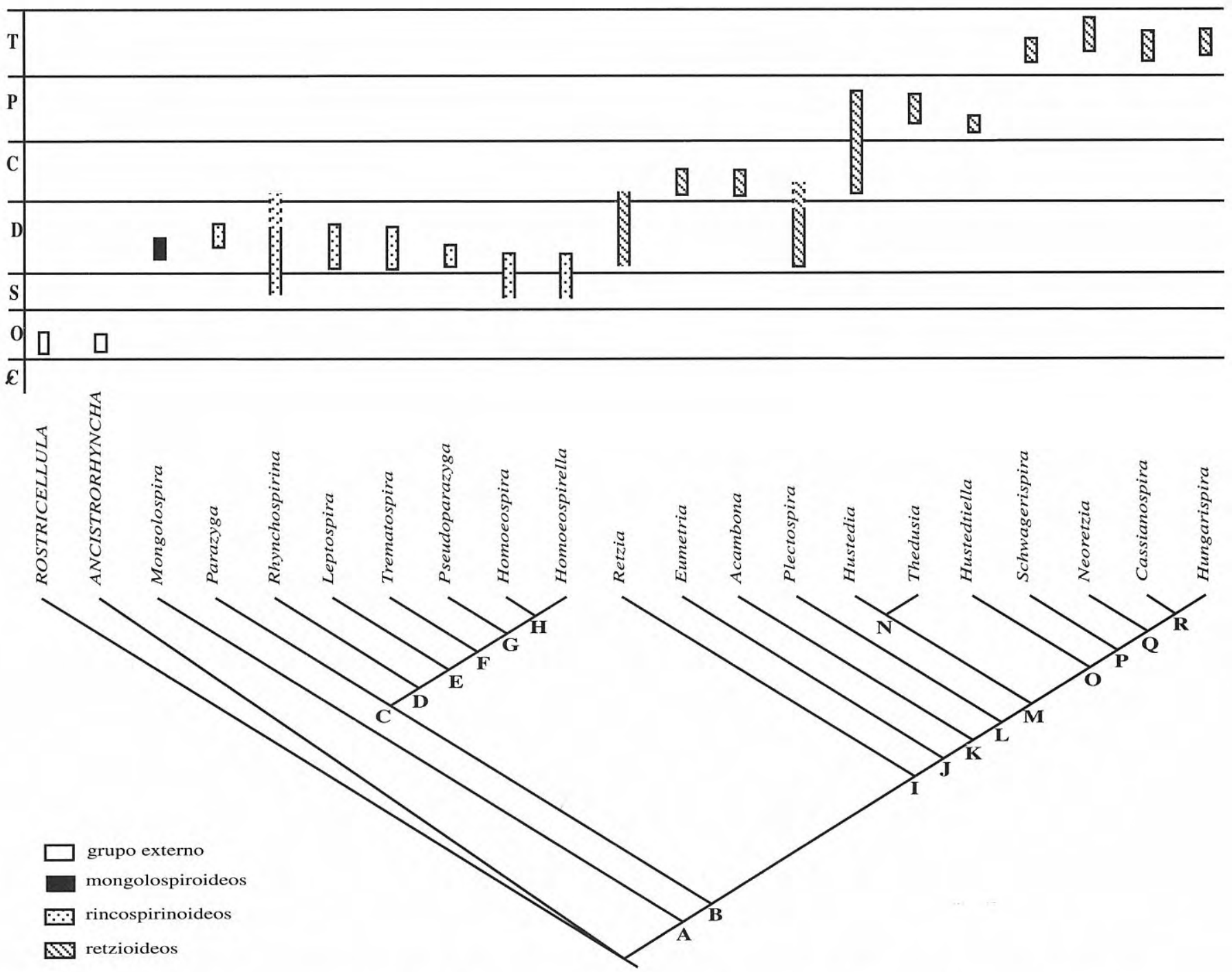

Figura 12. Cladograma número uno (uno de los 3, igualmente más parsimoniosos, resultantes en el séptimo análisis).

como prolongaciones directas de los crura, próximas y paralelas al plano sagital. Este tipo de braquidio es característico de los "espiriféridos" (espiriféridos y espiriferínidos).

Los braquiópodos con espiralio pero de concha astrófica desarrollaron, a diferencia con los "espiriféridos estróficos", dos tipos de espiralio más derivados. El primero, con los ápices dirigidos dorsalmente (dorsomedianamente en formas menos derivadas), con la lamela primaria curvándose lateralmente desde los crura y con procesos yugales, caracteriza a los atrípidos, grupo que apareció en el Ordovícico tardío (Ashgill) y se extinguió al final del Devónico Superior (Frasniense), desarrollándose especialmente bien en las familias más recientes.

Los lissatripidinos y los "atrípidos primitivos", aunque surgiendo primero (Llandeilo?-Caradoc) en el registro estratigráfico, en algunos análisis (comparar Figs. 1 y 5) parecen compartir caracteres morfológicamente más derivados que los atripidinos (Ashgill). Las lamelas primarias se curvan lateralmente desde los crura, con los extremos distales dirigidos bien hacia el plano sagital (raramente), o bien dorsal o dorsomedianamente. El yugo, cuando aparece completo, se sitúa anteriormente en las formas primitivas, desplazándose en dirección posterior en formas más recientes, pero manteniéndose siempre situado en la región dorsal, bajo los conos espiralados.

El segundo tipo se desarrolló en todos los grupos generalmente clasificados como atirididos s.l., los cuales forman un grupo monofilético en los análisis I, II y III. En este espiralio, las lamelas primarias se curvan posterodorsalmente desde las terminaciones distales de los crura, paralelamente al plano de simetría; los ápices de los conos de las espiras se dirigen lateralmente; desarrollan yugo completo, situado en la región ventral (entre los conos de las espiras). Las ramas laterales del yugo, que generalmente comienzan posteriormente a la mitad de la longitud de la valva dorsal, se inclinan en dirección posterior en formas primitivas y son verticales o se inclinan anteriormente en géneros más recientes. El arco yugal, agudo en algunas formas primitivas (e.g. nucleospiroideos y algunos meristéllidos) y redondeado en taxones más derivados (atiridoideos, retziidinos, 
retzielloideos y algunos meristelloideos), puede proyectarse anteriormente en una visera más o menos prominente (algunos retziellidinos presentan viseras similares pero dirigidas en dirección posterior); prácticamente todas las formas avanzadas (atiridoideos, retzioideos y algunos meristelloideos) poseen tallo yugal; las lamelas yugales accesorias, cuando se presentan, son generalmente cortas o terminan en las proximidades del inicio dorsal de las ramas laterales del yugo (atiridoideos primitivos). En algunas formas primitivas (algunos meristelloideos), se unen a las ramas laterales o a la base del tallo; y en algunas formas derivadas conectan secundariamente con la primera espira del braquidio, en su mitad ventral (neoretziidos triásicos), o se prolongan intercalándose con los conos espiralados principales hasta sus extremos distales (diplospirellinos, más Helenathyris y Kayseria).

Los koninckinoideos aparecen (Fig. 1, ver también las Figs. 3 y 4) parafiléticamente junto con lissatripidinos y "atrípidos primitivos" en la base de los grupos clasificados frecuentemente como atiridos s.l. Los koninckinoideos se caracterizan por su perfil lateral plano o concavoconvexo, casi estróficos, espiralio con los ápices dirigidos ventralmente y con lamelas accesorias libres intercaladas con las espiras principales hasta el final. Esta orientación de los conos de espiras, junto con su perfil lateral y su carácter "casi" estrófico se aleja mucho de las características presentes en otros grupos de braquiópodos con espiralio y hacen problemática su clasificación ordinal.

\section{SUMARIO Y CONCLUSIONES}

1. Las relaciones filogenéticas entre los taxones de alto rango entre los "braquiópodos articulados" (ver discusión en Williams et al., 1996), en particular de aquellos que desarrollaron un espiralio como soporte mineralizado del lofóforo, y las clasificaciones resultantes no son universalmente aceptadas debido especialmente al modelo complejo de evolución dentro del filo. Los resultados presentados en este trabajo, usando metodología filogenética en conjunción con métodos tradicionales, muestran una imagen de esta evolución, más completa y fácilmente verificable que la obtenida por cualquiera de ellos utilizado por separado. Los resultados de 8 análisis filogenéticos experimentales, seleccionados entre un amplio conjunto de ensayos, usando grupos externos para la determinación de la polaridad son comparados y contrastados. Se discute la evolución de los caracteres morfológicos dentro de y entre los grupos y se realiza una comparación entre los métodos estratigráficos (criterio paleontológico) y aquellos que utilizan grupos externos para la determinación de la polaridad en los cambios. Se espera que las hipótesis filogenéticas obtenidas en estos análisis sirvan de base para un mejor conocimiento de las tendencias evolutivas entre los braquiópodos con braquidio espiralado en general y los atíridos en particular.

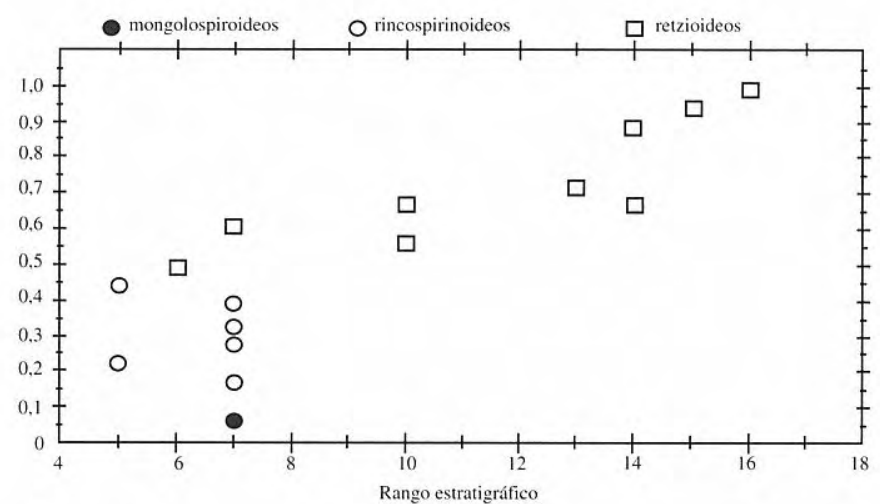

Figura 13. Representación del rango cladístico frente al rango estratigráfico.

2. Información estratigráfica (datos de primeras apariciones) y morfológica (rango cladístico) fueron combinados en un análisis único y los resultados comparados con diagramas filogenéticos establecidos independientemente de la posición estratigráfica de los taxones para así comprobar si era congruente la información obtenida con ambos criterios de polaridad. En los resultados obtenidos, existe una clara correlación positiva entre la primera aparición de los taxones y el rango cladístico, lo que corrobora la congruencia entre ambos criterios de polaridad.

3. Los grupos principales de "braquiópodos articulados", tal como tradicionalmente eran entendidos, aparecen como parafiléticos. Los braquiópodos estróficos, con espiralio, los rinconélidos, pentaméridos, terebratúlidos, los braquiópodos astróficos, con espiralio, atrípidos, koninckinidinos, atíridos s.l., retzielloideos, meristelloideos y retziidinos son cada uno de ellos monofilético con respecto a los resultados descritos en este trabajo (Figs. 1, 3, 4 y 5).

4. En general, los atiridoideos, meristelloideos, retziidinos y retzielloideos, mantienen su identidad en los diferentes ensayos realizados, siendo la organización interna bastante constante, fuese cual fuere la combinación grupo externo/grupo interno utilizada, si bien su posición en el árbol, y sus relaciones recíprocas pueden variar de un análisis a otro.

5. Spiriferida, tal como fue definido por Boucot et al. (1965), es un grupo polifilético. En las hipótesis de trabajo aquí propuestas sobre las relaciones filogenéticas (Figs. 1, 3, 4), los braquiópodos estróficos con espiralio retienen más características primitivas que los rinconélidos, pentaméridos o los braquiópodos astróficos con espiralio. Rinconélidos y pentaméridos retienen algunos caracteres primitivos pero poseen también caracteres derivados que los distinguen, como clados, de sus antecesores. Esta es también la situación común entre los grupos de braquiópodos astróficos con espiralio, ramificando entre pentaméridos y retziidinos (el grupo más derivado en el análisis) (Fig. 1).

6. El análisis cladístico soporta anteriores sugerencias de que los braquiópodos estróficos con espiralio ("espiriféridos") constituyen un clado separado del resto 


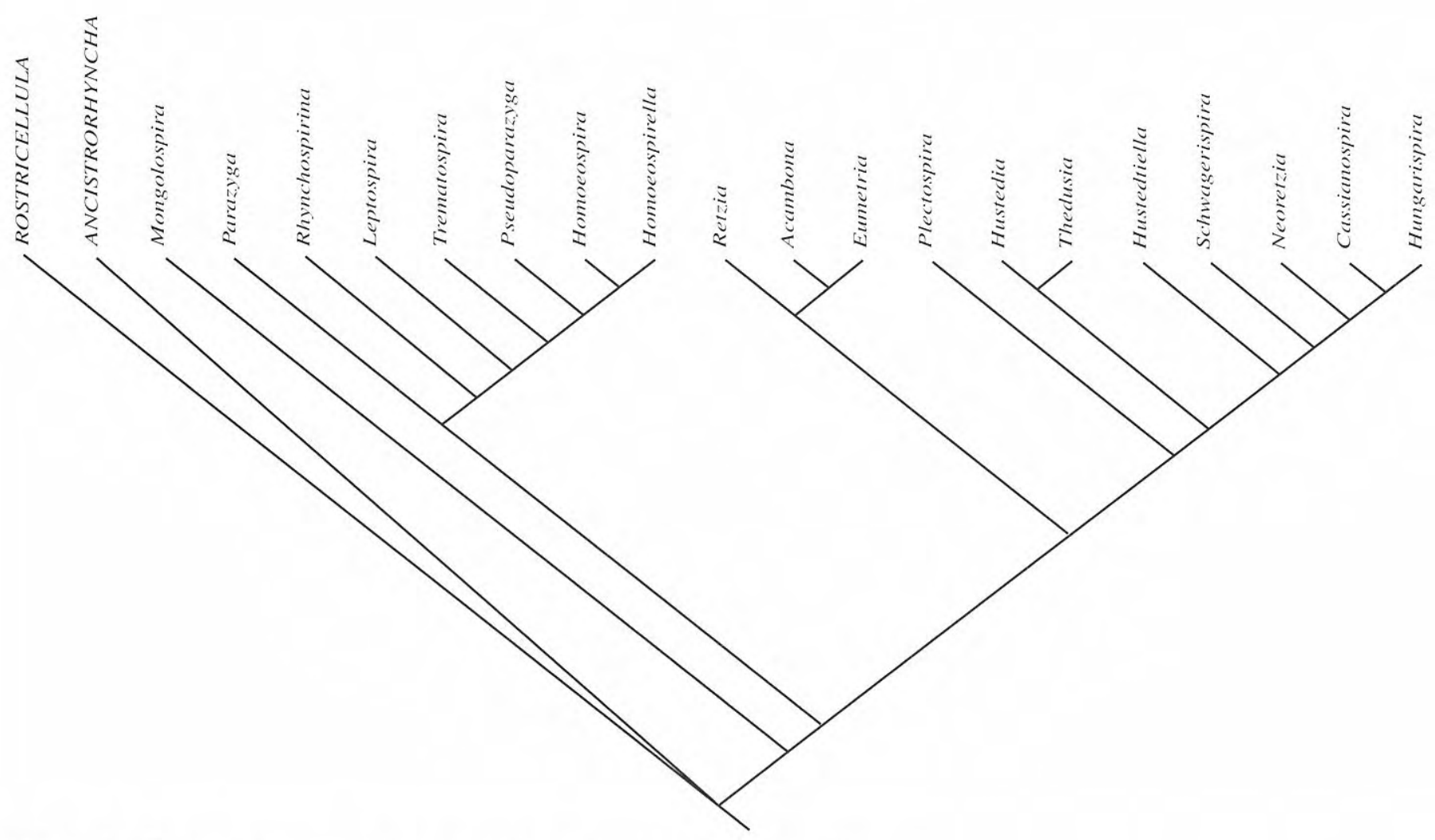

Figura 14. Único cladograma, más parsimonioso, obtenido del octavo análisis).

de braquiópodos con espiralio pero astróficos. Espiriféridos puntuados (Spiriferida) e impuntuados (Spiriferinida) constituyen un grupo bien definido en la base del cladograma, lejos de los braquiópodos astróficos con espiralio. Parece pues que la calcificación de un espiralio, como soporte rígido para el lofóforo, no indica necesariamente una relación directa, ya que este hecho pudo haber ocurrido en diferentes ramas filéticas (e.g. Thomson, 1925; Ivanova, 1959; Rzhonsnitskaia, 1960; Rudwick, 1970; Wright, 1979a, b; Carter et al., 1994). A pesar de su aparición relativamente tardía, los "espiriféridos" parecen ser, morfológicamente, los braquiópodos con espiralio más primitivos (ver las Figs. 1,3 y 4), lo que excluiría su posible derivación tanto de los atrípidos como de los atíridos.

7. Los cladogramas (Figs. 1, 3, 4) ordenan los atrípidos como grupo hermano de los primeros braquiópodos astróficos que desarrollaron espiralio, los cuales parecen poseer caracteres más derivados. Los Dayiinae, clasificados en el Treatise (Boucot et al., 1965, ver también Johnson, 1970) como atripidinos pero reasignados a los Athyrididina por Copper (1973a, b, 1986a), en nuestros análisis se agrupan constantemente con los lissatripidinos (Caradoc-Givetiense), situándose entre los braquiópodos astróficos con espiralio, costados (carácter primitivo) y los lisos (derivado) (Fig. 1, ver también las Figs. 3, 4 y 5). Los koninckinoideos se presentan parafiléticamente en la base de los grupos frecuentemente clasificados como atíridos s.l. La orientación ventral del ápice de los conos de espiras, junto con su perfil lateral característico y su carácter casi estrófico los aleja del resto de braquiópodos con espiralio. Estos caracteres morfológicos peculiares, junto con su distribución estratigráfica, hacen problemática su clasificación ordinal. El resto de braquiópodos astróficos con espiralio, es decir, todos los grupos frecuentemente clasificados como atíridos s.l., forman un grupo monofilético. Los cambios más importantes se producen en el espiralio (ver el subapartado braquidio dentro del apartado DISCUSIÓN). Los retzielloideos, aparecen generalmente en la base del clado, frecuentemente como grupo hermano con Bifida y Kayseria. No obstante, muestran un comportamiento inestable dependiendo de los grupos seleccionados para la matriz (grupos interno y externo) (Figs. 1, 3, 5). En el análisis I (Fig. 1) los retzielloideos son grupo hermano de los meristelloideos, mientras que, por ejemplo, en el análisis II (Fig. 4), se desplazan hasta el final del cladograma como grupo hermano de los retziidinos. Este comportamiento, junto con su morfología externa e interna (e.g. Rong et al., 1994) particular y su distribución geográfica y estratigráfica hacen que su afinidad y rango sistemático sean inciertos.

8. Con los meristelloideos, la concordancia entre los datos de primera aparición de los grupos principales y su rango cladístico es muy buena (ver la Fig. 8). Los meristelloideos primitivos forman un clado en la base de los meristéllidos; los merístidos forman un grupo bien definido, compartiendo los caracteres más derivados, en el que Meristinae y Camarophorellinae aparecen como grupos hermanos. Los atiridoideos más primitivos generalmente aparecen parafiléticamente en la base del 
clado. La mayor parte de los atíridos "lisos" muestran una gran politomía entre estas formas primitivas y aquellas con ornamentación radial, las cuales fueron agrupadas en el extremo distal de los cladogramas obtenidos en el análisis IV. Al pesar dos caracteres relacionados con el desarrollo del cardinalio dorsal, el modelo de relación entre grupos que surge (Fig. 10) es bastante próximo a las filogenias sugeridas empleando sólo métodos tradicionales. La comparación de los datos de primera aparición con el rango cladístico obtenido en este análisis indica una correlación positiva entre ambas variables muy buena, lo que soporta, por tanto, las relaciones filogenéticas sugeridas.

9. Los atirisininos, que generalmente fueron clasificados como una superfamilia entre los retziidinos (e.g. Boucot et al., 1965), se agrupan constantemente con los atiridoideos, entre los atiridinos y los plicatiridinos. Por tanto, los atirisininos deben ser incluidos entre los atiridoideos. El análisis muestra también que atirididos con espinas macizas (e.g. Cleiothyridina) y los lonchengiinos se encuentran estrechamente relacionados con los atirididos no costados. Atíridos con caracteres más derivados, como son la plataforma cardinal no perforada o el enorme desarrollo de rebordes cardinales (cardinal flanges), forman constantemente un clado en la parte superior del cladograma.

10. La concordancia entre los datos de primera aparición de los grupos principales de retziidinos y su rango cladístico es muy buena, lo que sugiere que tanto los métodos tradicionales (e.g. Dagis, 1974; Grunt, 1986) como los que utilizan un grupo externo (Fig. 12) indican una dirección similar en la polaridad de los cambios evolutivos en los retziidinos (ver también las Figs. 13 y 14). Tres grupos de géneros son fácilmente reconocibles. En la base del cladograma aparecen los mongolospiroideos, grupo extraño de concha lisa. Los rincospirinoideos agrupados en un clado, bien resuelto, caracterizado por la posesión de una plataforma cardinal con las placas cardinales internas poco, o nada, desarrolladas. Espinas huecas, largas y numerosas, dirigidas en dirección anterior o anterolateral caracterizan a los parazygidos, que aparecen como antecesores de los demás rincospirinoideos. En la parte superior del cladograma aparecen los retzioideos, tipificados por poseer un plataforma cardinal no perforada con los rebordes cardinales (cardinal flanges) moderada o fuertemente desarrollados y con el yugo carente de cualquier tipo de visera pero con un tallo largo dividido, en los géneros más recientes, en más o menos elaboradas lamelas yugales accesorias. Neoretziidos, más derivados, poseen una concha prácticamente estrófica, con el gancho ventral casi recto e internamente con las lamelas yugales accesorias pudiendo unirse secundariamente al espiralio o permanecer libres e intercalarse entre los conos principales hasta su ápice.

11. En los análisis, los retziidinos no se agrupan nunca con los espiriferinidos (braquiópodos estróficos, con concha puntuada y con espiralio), tal como sugirieron Cooper y Grant (1976) y Carter (1990), sino que aparecen siempre como el subclado más derivado dentro del grupo de los atíridos. En los análisis filogenéticos, cada subclado puede ser considerado como un taxón nuevo, lo que puede provocar una enorme proliferación de nombres nuevos, hecho que acarrea el efecto contrario al que inicialmente se pretendía, la clarificación del grupo estudiado (ver Queiroz y Gauthier, 1990; Smith, 1994). En el caso de los retziidinos, se ha preferido considerarlos como un suborden (el más "evolucionado" de los atíridos), en lugar de orden (cf. Waterhouse, 1981). Este proceder minimiza el número de grupos parafiléticos nombrados en el proceso.

12. De acuerdo con los resultados obtenidos en los diferentes análisis, la aparición de charnelas prácticamente estróficas, foramen transapical, o de estructuras internas tales como el espondilio, shoe-lifter, cruralio, septalio, plataformas cardinales de tipo diverso, espiralio y yugos más o menos elaborados, así como la condición puntuada de la concha, se produjo independientemente, en diferentes momentos y en diferentes grupos de "braquiópodos articulados".

13. Los diferentes análisis realizados muestran las ventajas y los riesgos que el análisis cladístico lleva consigo. Parece claro, como ocurre con cualquier análisis estadístico, que se pueden obtener distintos resultados o conclusiones con sólo introducir cambios menores en los datos de partida. Si se quiere que los análisis cladísticos tengan un valor filogenético, éstos han de realizarse con grupos sobre los que se posea una abundante información acerca de su morfología, anatomía, estructura, variabilidad, ontogenia y distribución temporal y espacial. En caso contrario, podemos caer en el peligro de llegar a conclusiones o escoger alternativas cuando menos arriesgadas.

\section{AGRADECIMIENTOS}

Los autores agradecen a numerosos colegas, en particular Alwyn Williams, C. Howard C. Brunton, Rong Jia-yu, Arthur, J. Boucot, Tania L. Modzalevskaia y Rex Doescher, sus respuestas y comentarios a determinados puntos expresados en este trabajo. A Covadonga Brime por su constante y desinteresada ayuda y a $\mathrm{M}^{\mathrm{a}}$ Luisa Martínez Chacón y a los revisores del manuscrito (Arthur, J. Boucot, C. Howard C. Brunton y Nieves López-Martínez) por su lectura crítica y las sugerencias realizadas.

\section{BIBLIOGRAFÍA}

Alekseeva, R.E. 1977. Novyi rod rettsiidnykh brakhiopod. [Un género nuevo de braquiópodos retzios]. In: Bespozvonochnye Palozoia Mongolii. Sovmestnaia Sovetsko-Mongol'skaia Paleontologicheskaia Ekspeditsiia (SSMPE), [Invertebrados paleozoicos de Mongolia: expedición paleontológica conjunta Mongolo-soviética] (Eds. L.P. Tatarinov, B. Luvsandansan, J.I. Voronin, R. Barsbold, I.P. Morozova \& G.A. Afana'sieva). Trudy (Moscow), 63-65. 
Álvarez, F. 1990. Devonian athyrid brachiopods from the Cantabrian Zone (NW Spain). Biostratrigraphie du Paléozoique, 11, 1-311.

Álvarez, F. \& Brunton, C.H.C. 1990a. Pachyplax n.g. (Brachiopoda, Athyridacea) from the Lower Devonian of the Cantabrian Mountains, NW Spain. Senckenbergiana lethaea, 70, 29-50.

Álvarez, F. \& Brunton, C.H.C. 1990b. The shell-structure, growth and functional morphology of some Lower Devonian athyrids from northwest Spain. Lethaia, 23, 117-131.

Álvarez, F. \& Brunton, C.H.C. 1995. Rowleyellinae, a new subfamily of Lower Carboniferous meristelloid athyridid brachiopods, endemic to North America. Journal of Paleontology, 69, 605-606.

Álvarez, F., MacKinnon, D.I. \& Rong Jia-yu. 1994. [Compilation of Annual Reports (1993-1994). Treatise on Invertebrate Paleontology, Part $H$, revision. Unpublished report].

Álvarez, F., Rong Jia-yu \& Boucot, A.J. (en prensa). The classification of athyridid brachiopods. Journal of Paleontology.

Boucot, A.J. \& Wilson, R.A. 1994. Origin and early radiation of terebratuloid brachiopods: thoughts provoked by Prorensselaeria and Nassothyris. Journal of Paleontology, 68, 1002-1025.

Boucot, A.J., Johnson, J.G., Pitrat, C.W. \& Staton, R.D. 1965. Order Spiriferida Waagen, 1883. In: Treatise on Invertebrate Paleontology, Part H. Brachiopoda. (Ed. R.C. Moore). Geological Society of America and University of Kansas Press, Boulder, Colorado and Lawrence, Kansas, H632-H728.

Brunton, C.H.C., Álvarez, F. \& MacKinnon, D.I. 1996. Morphological terms used to describe the cardinalia of articulate brachiopods: homologies and recommendations. Historical Biology, 11, 9-41.

Carlson, S.J. 1989. The articulate brachiopod hinge mechanism: morphological and functional variation. Paleobiology, 15, 364-386.

Carlson, S.J. 1991. Phylogenetic relationships among brachiopod higher taxa. In: Brachiopods through time (Eds. D.I. MacKinnon, D.E. Lee \& J.D. Campbell). A.A. Balkema, Rotterdam, Netherlands, 3-10.

Carlson, S.J. 1992. Evolutionary trends in the articulate brachiopod hinge mechanism. Paleobiology, 18, 344366.

Carlson, S.J. 1993. Phylogeny and evolution of 'pentameride' brachiopods. Palaeontology, 36, 807-837.

Carlson, S.J. 1995. Phylogenetic relationships among extant brachiopods. Cladistics, 11, 131-197.

Carter, J.L. 1990. New brachiopods (Brachiopoda: Articulata) from the Late Osagean of the Upper Mississippi Valley. Annals of Carnegie Museum, 59, 219-247.

Carter, J.L., Johnson, J.G., Gourvennec, R. \& Hou Hongfei. 1994. A revised classification of the spiriferid brachiopods. Annals of Carnegie Museum, 63, 327-374.

Cocks, L.R.M. 1978. A review of British Lower Palaeozoic brachiopods, including a synoptic revision of Davidson's Monograph. Palaeontographical Society Monograph,
131(549), 1-256.

Cooper, G.A. \& Grant, R.E. 1976. Permian brachiopods of West Texas, V. Smithsonian Contributions to Paleobiology, 24, 2609-3159.

Copper, P. 1973a. Bifida and Kayseria (Brachiopoda) and their affinity. Palaeontology, 16, 117-138.

Copper, P. 1973b. New Siluro-Devonian atrypoid brachiopods. Journal of Paleontology, 47, 484-500.

Copper, P. 1986a. Filter-feeding and evolution in earlyspire-bearing brachiopods. In: Les Brachiopodes fossiles et actuels (Eds. P.R. Racheboeuf \& C.C. Emig). Biostratigraphie du Paléozoique, 4, 219-230.

Copper, P. 1986b. Evolution of the earliest smooth spirebearing atrypoids (Brachiopoda: Lissatrypidae, Ordovician-Silurian). Palaeontology, 29, 827-866.

Copper, P. 1991. Evolution of atrypid brachiopods. In: Brachiopods through time (Eds. D.I. MacKinnon, D.E. Lee \& J.D. Campbell). A.A. Balkema, Rotterdam, Netherlands, 35-40.

Copper, P. 1996. Davidsonia and Rugodavidsonia (new genus), cryptic Devonian atrypid brachiopods from Europe and South China. Journal of Paleontology, 70, 588-602.

Copper, P. \& Gourvennec. R.1996. Evolution of the spirebearing brachiopods (Ordovician-Jurassic). In: Brachiopods (Eds. P. Copper \& J. Jin). A.A. Balkema, Rotterdam, Netherlands, 81-88.

Dagis, A.S. 1974. Triasovye brakhiopody (morfologiia, sistema, filogeniia, stratigraficheskoe znachenie $i$ biogeografiia). [Braquiópodos triásicos (morfología, clasificación, filogenia, significado estratigráfico y biogeografía).] Academiia Nauk SSR, Sibirskoe Otdelenie, Trudy Instituta Geologii i Geofiziki, 214, 1387.

Dagis, A. 1996a. On the classification of the order Athyridida. In: Brachiopods (Eds. P. Copper \& J. Jin). A.A. Balkema, Rotterdam, Netherlands, 89-90.

Dagis, A. 1996b. Remarks on the classification of punctate spiriferids. In: Brachiopods (Eds. P. Copper \& J. Jin). A.A. Balkema, Rotterdam, Netherlands, 91-93.

Davidson, T. 1881. On genera and species of spiral-bearing Brachiopoda, from specimens developed by the Rev. Norman Glass. Geological Magazine, 8(4), 1-13.

Forey, P.L. 1987. The Downtonian ostracoderm Sclerodus Agassiz (Osteostraci: Tremataspididae). Bulletin of the British Museum (Natural History), Geology, 41, 1-29.

Fortey, R.A. \& Chatterton, B.D.E. 1988. Classification of the trilobite suborder Asaphina. Palaeontology, 31, 165222.

Gauthier, J.A. 1986. Saurichian monophyly and the origin of birds. In: The origin of birs and the evolution of flight (Ed. K. Padian). Memoirs of the California Academy of Science, 8, 1-55.

Gauthier, J.A., Kluge, A.G. \& Rowe, T. 1988. Amniote phylogeny and the importance of fossils. Cladistics, $\mathbf{4}$, 105-209.

Grunt, T.A. 1986. Sistema brakiopod otriada Atiridida. [Clasificación de los braquiópodos del orden Athyridida]. Academiia Nauka USSR, Trudy Paleontologicheskogo Instituta, 215, 1-200. 
Grunt, T.A. 1989. Otriad Atiridida (evoliutsionnaia morfologiia $i$ istoricheskoe razvitie). [Orden Athyridida (morfología evolutiva y filogenia]. Academiia Nauka USSR, Trudy Paleontologicheskogo Instituta, 238, 1141.

Hall, J. \& Clarke, J.M. 1894. An introduction to the study of the Brachiopoda, intended as a handbook for the use of students, Part II. New York State Geologist, Annual Report, 13, 751-943.

Harper, D.A.T., Brunton, C.H.C., Cocks, L.R.M., Copper, P., Doyle, E.N., Jeffrey, A.L., Owen, E.F., Parkes, M.A., Popov, L.E. \& Prosser, C.D.1993. Brachiopoda. In: The Fossil Record 2 (Ed. M. J. Benton). Chapman and Hall, London, 430-462.

Havlicek, V. 1961. Rhynchonelloidea des böhmischen älteren Paläozoikums (Brachiopoda). Rozpravy Ustredniho ustavu gelogickeho, 27, 1-211.

Holmer, L.E., Popov, L.E., Bassett, M.G. \& Laurie, J. 1995. Phylogenetic analysis and ordinal classification of the brachiopoda. Palaeontology, 38, 713-741.

Ivanova, E.A. 1959. K sistematike i evoliutsii spiriferid (Brachiopoda) [Sobre la sistemática y evolución de los espiriféridos (Brachiopoda)]. Paleontologicheskii Zhurnal, 4, 47-64.

Ivanova, E.A. 1960. Otriad Spiriferida. [Orden Spiriferida.] In: Osnovy paleontologii: Spravochnik dlia paleontologov i geologov SSSR. Mshanki, Brakhiopody [Principios de Paleontología: Un manual para los paleontólogos y geólogos de la URSS. Briozoos, braquiópodos] (Ed. T. G. Sarycheva). Izdatel'stvo Akademii Nauk SSSR, Moskva, 264-280.

Jaanusson, V. 1971. Evolution of the brachiopod hinge. In: Paleozoic Perspectives: A Paleontological Tribute to G. Arthur Cooper (Ed. J.T. Jr. Dutro). Smithsonian Contributions to Paleobiology, 3, 33-46.

Jin, J., Caldwell, W.G.E. \& Norford, B.S. 1993. Early Silurian brachiopods and biostratigraphy of the Hudson Bay Lowlands, Manitoba, Ontario, and Quebec. Geological Survey of Canada Bulletin, 457, 1-221.

Johnson, J.G. 1970. Great Basin Lower Devonian Brachiopoda. The Geological Society of America, Memoire, 121, 1-421.

King, W. 1846. Remarks on certain genera belonging to the class Palliobranchiata. Annals and Magazine of Natural History, 18, 26-42, 83-94.

Klassen, G.J., Mooi, R.D. \& Locke, A. 1991. Consistency indices and random data. Systematic Zoology, 40, 446457.

Likharev, B.K., Makridin, B.P., Nikiforova, O.I. \& Rzhonsnitskaia, M.A. 1960. Nadsemistvo Athyracea [Supefamilia Athyracea.]. In: Osnovy paleontologii: Spravochnik dlia paleontologov $i$ geologov SSSR. Mshanki, Brakhiopody [Principios de Paleontología: Un manual para los paleontólogos y geólogos de la URSS. Briozoos, braquiópodos] (Ed. T.G. Sarycheva). Izdatel'stvo Akademii Nauk SSSR, Moskva, 280-285.

Maddison, W.P. \& Maddison, D.R. 1992. MacClade: analysis of phylogeny and character evolution, version 3.0. Sunderland, Massachusetts: Sinauer Associates Ltd.

Muir-Wood, H.M. 1955. A history of the classification of the phylum Brachiopoda. British Museum (Natural History), London. 124 pp.

Queiroz, K. de \& Gauthier, J. 1990. Phylogeny as a central principle in taxonomy: phylogenetic definitions of taxon names. Systematic Zoology, 39, 307-322.

Rong Jia-yu, \& Zhan Ren-bin. 1996. Brachidia of Late Ordovician and Silurian eospiriferines (Brächiopoda) and the origin of the spiriferides. Palaeontology, 39, 941977.

Rong Jia-yu, Zhang Yan \& Chen Xiu-quin. 1987. Braquiópodos pridolienses y lochkovienses del área de Luqu-Tewo en las Montañas Qinling occidentales, China. In: Estratigrafía y paleontología del Silúrico tardío - Devónico del área de Luqu-Tewo en las Montañas Qinling occidentales, China. Volumen 2, editado por el Instituto de Geología y Recursos Minerales de Xi'an y el Instituto de Geología y Paleontología de Nanjing, Academia Sinica, Nanjing: Nanjing University Press, 1-94. (En chino).

Rong Jia-yu, Strusz, D.L., Boucot, A.J., Fu Li-pu, Modzalevskaia, T.L. \& Su Yan-zheng. 1994. The Retziellidae (Silurian ribbed impunctate athyrididine brachiopods). Acta Paleontologica Sinica, 33, 545-574.

Rudwick, M.J.S. 1970. Living and fossil brachiopods. Hutchinson University Library, London, 199 pp.

Rzhonsnitskaia, M.A. 1960. Otriad Atrypida [Orden Atrypida.]. In: Osnovy paleontologii: Spravochnik dlia paleontologov i geologov SSSR. Mshanki, Brakhiopody [Principios de Paleontologáa: Un manual para los paleontólogos y geólogos de la URSS. Briozoos, braquiópodos] (Ed. T. G. Sarycheva). Izdatel'stvo Akademii Nauk SSSR, Moskva, 257-264.

Sanchiz, F.B. y Valdecasas, A.G. 1980. Criterios metodológicos y glosario español de términos utilizados en sistemática cladística. Boletín de la Real Sociedad Española de Historia Natural (Biología), 78, 223-244.

Sanderson, M.J. \& Donoghue, M.J. 1989. Patterns of variation in levels of homoplasy. Evolution, 43, 17811795.

Schuchert, C. 1896. Class 2. Brachiopoda. In: Text-book of palaeontology (Zittel, K.A. v., edited by Charles R. Eastman), 1, London, 291-343.

Schuchert, C. 1929. Classification of brachiopod genera, fossil and Recent. In: Brachiopoda (Generum et Genotyporum Index et Bibliographia). Fossilium Catalogus. I. Animalia, pt. 42 (C. Schuchert \& C.M. LeVene). W. Junk, Berlin, 10-25.

Smith, A.B. 1994. Systematics and the fossil record: documenting evolutionary patterns. Blackwell Scientific Publications, Oxford, 223 pp.

Swofford, D. L. 1990. PAUP(Phylogenetic Analysis Using Parsimony), versión 3.0q., Illinois Natural History Survey, 607 East Peabody Drive, Champaign, Illinois 61820.

Thomson, J.A. 1925. The interrelations of the superfamilies of the Telotremata (Brachiopoda). Annals and Magazine of Natural History, 16, 425-432.

Waagen, W.H. 1883. Salt Range fossils. ProductusLimestone fossils: Brachiopoda. Palaeontologica Indica, Ser. 13, 4(2), 391-546. 
Waterhouse, J.B. 1981. Early Permian brachiopods from Ko Yao Noi and near Krabi, Southern Thailand. In: The Permian stratigraphy and palaeontology of southern Thailand (J.B. Waterhouse, K. Pitakpaivan \& N. Mantajit). Memoir of the Geological Survey of Thailand, 4, 43-213.

Westbroek, P. 1967. Morphological observations with systematic implications on some Palaeozoic Rhynchonellida from Europe, with special emphasis on the Uncinulidae. Leidse Geologische Mededelingen, 41, $1-82$.

Westbroek, P. 1969. The interpretation of growth and form in serial sections through brachiopods, exemplified by the trigonirhynchiid septalium. Palaeontology, 12, 321332.

Wiley, E.O. 1981. Phylogenetics, the theory and practice of phylogenetic systematics. John Wiley \& Sons, New York, 439 pp.

Williams, A. \& Brunton, C.H.C. 1993. Role of the shell structure in the classification of the orthotetidine brachiopods. Palaeontology, 36, 931-966.

Williams, A. \& Hurst, J.M. 1977. Brachiopod evolution. In: Patterns of Evolution as Illustrated by the Fossil Record (Ed. A. Hallam). Elsevier, Amsterdam, 79-121.

Williams, A. \& Rowell, A.J. 1965. Evolution and phylogeny. In: Treatise on Invertebrate Paleontology, Part H, Brachiopoda (Ed. R.C. Moore). Geological Society of America and University of Kansas Press,
Boulder, Colorado and Lawrence, Kansas, H164-H197. Williams, A. \& Wright, A.D. 1965. Suborder Orthidina Schuchert \& Cooper, 1932. In: Treatise on Invertebrate Paleontology, Part H, Brachiopoda (Ed. R.C. Moore). Geological Society of America and University of Kansas Press, Boulder, Colorado and Lawrence, Kansas, H300H346.

Williams, A., Rowell, A.J., Muir-Wood, H.M., Pitrat, C.W., Schmidt, H., Stehli, F.G., Ager, D.V., Wright, A.D., Elliott, G.F., Amsden, T.W., Rudwick, M.J.S., Hatai, K., Biernat, G., Mclaren, D.J., Boucot, A.J., Johnson, J.G., Staton, R.D., Grant, R.E. \& Jope, H.M. 1965. Brachiopoda. In: Treatise on Invertebrate Paleontology, Part H, Brachiopoda (Ed. R.C. Moore). Geological Society of America and University of Kansas Press, Boulder, Colorado and Lawrence, Kansas, H1-H927.

Williams, A., Carlson, S.J., Brunton, C.H.C., Holmer L.E. \& Popov, L. 1996. A supra-ordinal classification of the Brachiopoda. Philosophical Transactions of the Royal Society of London, Series B, 351, 1171-1193.

Wright, A.D. 1979a. The origin of the spiriferidine brachiopods. Lethaia, 12, 29-33.

Wright, A.D. 1979b. Brachiopod radiation. In: The Origin of Major Invertebrate Groups (Ed. M.R. House). Academic Press, London, 235-252.

Zittel, K.A. v. 1903. Brachiopoda. In: Grundzüge der Paläontologie (Paläozoologie). I. Abteilung: Invertebrata., 2nd edit., München and Leipzig, 240-274. 